\title{
Dust grains from the heart of supernovae
}

\author{
M. Bocchio ${ }^{1,2}$, S. Marassi ${ }^{2}$, R. Schneider ${ }^{2}$, S. Bianchi ${ }^{1}$, M. Limongi ${ }^{2}$, and A. Chieffi ${ }^{3}$ \\ 1 INAF-Osservatorio Astrofisico di Arcetri, Largo Enrico Fermi 5, 50125 Firenze, Italy \\ e-mail: mbocchio@arcetri.astro.it \\ 2 INAF-Osservatorio Astronomico di Roma, via di Frascati 33, 00040 Monteporzio, Italy \\ 3 INAF/IASF, via Fosso del Cavaliere 100, 00133 Roma, Italy
}

Received 23 September 2015 / Accepted 13 January 2016

\begin{abstract}
Dust grains are classically thought to form in the winds of asymptotic giant branch (AGB) stars. However, there is increasing evidence today for dust formation in supernovae ( $\mathrm{SNe}$ ). To establish the relative importance of these two classes of stellar sources of dust, it is important to know the fraction of freshly formed dust in SN ejecta that is able to survive the passage of the reverse shock and be injected in the interstellar medium. With this aim, we have developed a new code, GRASH_Rev, that allows following the dynamics of dust grains in the shocked SN ejecta and computing the time evolution of the mass, composition, and size distribution of the grains. We considered four well-studied SNe in the Milky Way and Large Magellanic Cloud: SN 1987A, CasA, the Crab nebula, and N49. These sources have been observed with both Spitzer and Herschel, and the multiwavelength data allow a better assessment the mass of warm and cold dust associated with the ejecta. For each SN, we first identified the best explosion model, using the mass and metallicity of the progenitor star, the mass of ${ }^{56} \mathrm{Ni}$, the explosion energy, and the circumstellar medium density inferred from the data. We then ran a recently developed dust formation model to compute the properties of freshly formed dust. Starting from these input models, GRASH_Rev self-consistently follows the dynamics of the grains, considering the effects of the forward and reverse shock, and allows predicting the time evolution of the dust mass, composition, and size distribution in the shocked and unshocked regions of the ejecta. All the simulated models aagree well with observations. Our study suggests that SN 1987A is too young for the reverse shock to have affected the dust mass. Hence the observed dust mass of $0.7-0.9 M_{\odot}$ in this source can be safely considered as indicative of the mass of freshly formed dust in SN ejecta. Conversely, in the other three SNe, the reverse shock has already destroyed between $10-40 \%$ of the initial dust mass. However, the largest dust mass destruction is predicted to occur between $10^{3}$ and $10^{5}$ yr after the explosions. Since the oldest SN in the sample has an estimated age of $4800 \mathrm{yr}$, current observations can only provide an upper limit to the mass of SN dust that will enrich the interstellar medium, the so-called effective dust yields. We find that only between 1-8\% of the currently observed mass will survive, resulting in an average SN effective dust yield of $(1.55 \pm 1.48) \times 10^{-2} M_{\odot}$. This agrees well with the values adopted in chemical evolution models that consider the effect of the SN reverse shock. We discuss the astrophysical implications of our results for dust enrichment in local galaxies and at high redshift.
\end{abstract}

Key words. supernovae: general - dust, extinction - local insterstellar matter - galaxies: high-redshift

\section{Introduction}

It is observationally and theoretically well established that a considerable amount of dust is efficiently formed in regions around asymptotic giant branch (AGB) stars (e.g. Weigelt et al. 2002; Woitke 2006; Zhukovska et al. 2008; Olofsson et al. 2010; Schneider et al. 2014; Dell'Agli et al. 2015). This process is classically considered as the primary source of dust grains in galaxies (see however Schneider et al. 2014), and the typical formation timescale in the Milky Way is $\sim 3 \times 10^{9} \mathrm{yr}$ (Dwek \& Scalo 1980; Gehrz 1989; Jones \& Tielens 1994).

In contrast, supernova (SN) explosions in the interstellar medium (ISM) trigger shock waves that are able to quickly process dust grains and are considered the dominant mechanism of dust destruction in the ISM (Barlow 1978a,b; Draine \& Salpeter 1979a,b; Dwek \& Scalo 1980; Seab \& Shull 1983; McKee et al. 1987; Jones et al. 1994, 1996; Lakićević et al. 2015; Slavin et al. 2015). A recent theoretical work on interstellar dust destruction in shock waves led to an estimated lifetime of $\sim 6 \times 10^{7} \mathrm{yr}$ and $\sim 3 \times 10^{8} \mathrm{yr}$ for carbonaceous and silicate grains in our Galaxy, respectively (Bocchio et al. 2014), which is much shorter than the assumed dust formation timescale from AGB stars. This leads to the conclusion that a large amount of dust must be reaccreted from the gas phase. However, while there seem to be viable mechanisms for re-formation of carbonaceous grains under low temperature and pressure conditions (e.g. Dartois et al. 2005), silicate grains are harder to form under ISM conditions (Jones \& Nuth 2011).

Although $\mathrm{SNe}$ are believed to be efficient interstellar dust destroyers, there is increasing observational evidence today for the formation of non-negligible quantities of dust grains in the ejecta of SNe (e.g. Matsuura et al. 2009; Dunne et al. 2009; Gall et al. 2011; Gomez 2014). Given the relatively short timescale between the explosion of two $\mathrm{SNe}$ (estimated to be $\sim 40 \mathrm{yr}$ in our Galaxy, Li et al. 2011), this would lead to an effectively shorter timescale for dust formation.

Rapid dust enrichment is also required by millimeter (mm) and sub-millimeter (submm) observations of $z>6$ galaxies (Watson et al. 2015) and quasars (see Valiante et al. 2014 and references therein), which show that their ISM has already been enriched with $\gtrsim 10^{8} M_{\odot}$ of dust. Theoretical models and numerical simulations show that these large dust masses require efficient 
A\&A 587, A157 (2016)

Table 1. Observed and estimated physical properties of the four SNe compiled from the papers listed in column "Ref".

\begin{tabular}{|c|c|c|c|c|c|c|c|c|c|c|c|c|}
\hline SNR & Type & $\begin{array}{c}E_{\mathrm{ex}} \\
{\left[10^{51} \mathrm{erg}\right]}\end{array}$ & $\begin{array}{l}{ }^{56} \mathrm{Ni} \\
{\left[M_{\odot}\right]}\end{array}$ & $\begin{array}{l}M_{\text {prog }} \\
{\left[M_{\odot}\right]}\end{array}$ & $\begin{array}{c}\mathrm{Z} \\
{\left[Z_{\odot}\right]}\end{array}$ & $\begin{array}{c}\text { Age } \\
\text { [Years] }\end{array}$ & $\begin{array}{c}n_{0} \\
{\left[\mathrm{~cm}^{-3}\right]}\end{array}$ & $\begin{array}{l}M_{\text {dust }} \\
{\left[M_{\odot}\right]}\end{array}$ & Ref. & $\begin{array}{c}E_{\mathrm{ex}}^{\mathrm{mod}} \\
{\left[10^{51} \mathrm{erg}\right]}\end{array}$ & $\begin{array}{c}{ }^{56} \mathrm{Ni}^{\mathrm{mod}} \\
{\left[M_{\odot}\right]}\end{array}$ & $\begin{array}{l}M_{\mathrm{prog}}^{\mathrm{mod}} \\
{\left[M_{\odot}\right]}\end{array}$ \\
\hline 1987A & $\mathrm{II}^{a}$ & 1.1 & $0.075^{a}$ & $18-20^{a}$ & 0.4 & 27 & 1.5 & $0.7-0.9$ & $\mathrm{f}, \mathrm{g}, \mathrm{h}$ & 1.0 & 0.075 & 20 \\
\hline CasA & $\mathrm{IIb}^{b}$ & $1-3$ & $0.058-0.16^{c}$ & $13-20^{b}$ & 1 & 335 & 1.9 & $0.1-1$ & $\mathrm{i}, \mathrm{j}, \mathrm{k}$ & 1.5 & 0.11 & 20 \\
\hline Crab & IIn- $\mathrm{P}^{d}$ & 0.1 & $0.009-0.016^{d}$ & $9-12^{e}$ & 1 & 960 & 1 & $0.1-0.25$ & 1 & 0.5 & 0.014 & 13 \\
\hline N49 & II & 1.8 & N/A & $18-20$ & 0.4 & 4800 & 0.9 & $0.1-0.4$ & $\mathrm{~m}, \mathrm{n}$ & 1.5 & 0.075 & 20 \\
\hline
\end{tabular}

Notes. The last three columns indicate the input parameters used in the simulations.

References. ${ }^{(a)}$ Arnett et al. (1989); ${ }^{(b)}$ Krause et al. (2008); (c) Eriksen et al. (2009); (d) Smith (2013); (e) MacAlpine \& Satterfield (2008); ${ }^{(f)}$ Matsuura et al. (2011); ${ }^{\left({ }^{g}\right)}$ Indebetouw et al. (2014); ${ }^{(h)}$ Lakićević et al. (2011); ${ }^{(i)}$ Barlow et al. (2010); ${ }^{(j)}$ Rho et al. (2008); ${ }^{(k)}$ Dunne et al. (2009); ${ }^{(l)}$ Gomez et al. (2012); ${ }^{(m)}$ Park et al. (2012); ${ }^{\left({ }^{\prime}\right)}$ Otsuka et al. (2010).

grain growth in the dense phases of the ISM (Valiante et al. 2014; Michałowski 2015; Mancini et al. 2015), with both SN and AGB stars providing the first seed grains (Valiante et al. 2011).

One important source of uncertainty on the role of $\mathrm{SNe}$ as efficient dust polluters is whether the dust formed in the ejecta survives the passage of the reverse shock. The observed supernova remnants (SNR) are still relatively young (age $\lesssim 5000 \mathrm{yr}$ ), and the reverse shock has not reached the centre of the ejecta yet. The measured mass of dust associated with the ejecta will therefore not be entirely released into the ISM, but will be partly destroyed.

It has been possible to estimate the amount of dust in the ejecta in only four core-collapse $\mathrm{SNe}$ using observation from Spitzer and Herschel: SN 1987A, Cassiopeia A, the Crab nebula, and SNR N49. The photometer instruments on these two satellites, which together cover a wide range of wavelengths from the mid-infrared (MIR) through the far-infrared (FIR) and submm, are able to measure the emission from both cold and warm dust in $\mathrm{SNe}$. However, to estimate the dust mass responsible for the detected emission, correct assumptions must be made on the dust composition, size, and temperature distribution. Depending on the choice of the dust composition and on the size and temperature of the smallest grains, the dust mass estimate can vary by almost a factor of 6 (Mattsson et al. 2015). Furthermore, emission from cold dust in the molecular cloud surrounding a SN contaminates the emission from freshly formed dust in the ejecta, and different observations (e.g. polarisation measurements) are therefore needed to distinguish the two components (Dunne et al. 2009). The difficulty in determining how much emission comes from dust in the remnant and how much from the ISM was also recognised in the recent analysis of a sample of SNRs in the LMC by Lakićević et al. (2015): while they placed constraints on the influence of the remnant on the nearby ISM, the data lacked the resolution and sensitivity to study the amount of dust within the remnants themselves.

To estimate the dust mass that is released into the ISM after the passage of the reverse shock, a numerical code that simulates the dust dynamics and processing in $\mathrm{SNe}$ is needed. Using numerical simulations, Nozawa et al. $(2006,2007)$ estimated that depending on the energy of the explosion and on the density of the ISM, the fraction of dust surviving the reverse shock can vary between 0 and $80 \%$. Bianchi \& Schneider (2007, BS07) estimated that depending on the density of the surrounding interstellar medium, between 2 and $20 \%$ of the initial dust mass survives the passage of the reverse shock.

In this work we present a new code called GRASH_Rev, which treats dust processing in a supernova explosion. This code couples all the dust processing included in the GRASH_EX code (Bocchio et al. 2014) with the dynamics and structure of the SN as modelled by BS07, but extending it to include the full dynamics of dust grains within the ejecta and in the surrounding ISM.

This paper is organised as follows: in Sect. 2 we first present the SN sample that we have considered to compare the model predictions with observational data. In Sect. 3 we describe the corresponding SN explosion models and the properties of dust formed in their ejecta. In Sect. 4 we present the structure of the numerical model, the grid used in the calculation, how we describe gas and dust dynamics, and the physical processes included in dust processing. In Sects. 5 and 6 we present the main results and discuss their astrophysical implications. Finally, in Sect. 7 we draw our conclusions.

\section{Supernova sample}

We considered the four core-collapse SNRs: SN 1987A, Cassiopeia A, the Crab nebula, and SNR N49. In Table 1 we report some of their physical properties, as obtained from the literature: the type of $\mathrm{SN}$, the explosion energy $\left(E_{\mathrm{ex}}\right)$, the mass of ${ }^{56} \mathrm{Ni}$, the progenitor mass $\left(M_{\mathrm{prog}}\right)$, the estimated metallicity $(Z)$ of the progenitor star, the age, the number density of the circumstellar ISM $\left(n_{0}\right)$, and the measured dust mass associated with the ejecta $\left(M_{\text {dust }}\right)$. Some of these quantities are directly estimated from the data, while others are obtained by comparing the observed properties with theoretical models. For example, the progenitor mass and explosion energy of SN 1987A are obtained by fitting the observed light curve and spectrum with a theoretical model (Nomoto et al. 1994, 2006). Similarly, the mass of ${ }^{56} \mathrm{Ni}$ is estimated from comparison between theoretical and observed light curves. The uncertainties or approximations of the models are encoded in the final estimates of these physical quantities.

\section{1. $S N 1987 A$}

SN 1987A is a young SN located in the Large Magellanic Cloud (LMC). It exploded in 1987. Observational data in Table 1 are from Gomez (2013) and references therein. The dust mass estimate has been updated following the recent work by Matsuura et al. (2015), leading to a total dust mass of $\sim 0.8 M_{\odot}$. However, a recent and new analysis of the infrared emission from SN 1987A by Dwek \& Arendt (2015) points to a lower dust mass of $\sim 0.45 M_{\odot}$, mostly composed of silicate grains.

\subsection{Cassiopeia $A$}

Cassiopeia A (CasA) is a Galactic SNR resulting from a SN explosion that occurred 335 years ago. The kinetic energy has been estimated to be $\sim(2-3) \times 10^{51} \mathrm{erg}$ (Laming \& Hwang 2003). The 
Table 2. Dust distribution parameters and masses for the four $\mathrm{SNe}$ in the sample.

\begin{tabular}{|c|c|c|c|c|c|c|c|c|c|c|c|c|}
\hline & \multicolumn{3}{|c|}{ 1987A } & \multicolumn{3}{|c|}{ CasA } & \multicolumn{3}{|c|}{ Crab } & \multicolumn{3}{|c|}{ N49 } \\
\hline & $\begin{array}{c}a_{0} \\
(\mathrm{~nm})\end{array}$ & $\sigma$ & $\begin{array}{l}M_{\text {dust }} \\
\left(M_{\odot}\right)\end{array}$ & $\begin{array}{c}a_{0} \\
(\mathrm{~nm})\end{array}$ & $\sigma$ & $\begin{array}{l}M_{\text {dust }} \\
\left(M_{\odot}\right)\end{array}$ & $\begin{array}{c}a_{0} \\
(\mathrm{~nm})\end{array}$ & $\sigma$ & $\begin{array}{l}M_{\text {dust }} \\
\left(M_{\odot}\right)\end{array}$ & $\begin{array}{c}a_{0} \\
(\mathrm{~nm})\end{array}$ & $\sigma$ & $\begin{array}{l}M_{\text {dust }} \\
\left(M_{\odot}\right)\end{array}$ \\
\hline $\mathrm{Al}_{2} \mathrm{O}_{3}$ & 7.5 & 0.14 & $2.57 \times 10^{-2}$ & 5.5 & 0.14 & $2.57 \times 10^{-2}$ & 4.7 & 0.01 & $5.18 \times 10^{-3}$ & 5.5 & 0.14 & $2.57 \times 10^{-2}$ \\
\hline $\mathrm{Fe}_{3} \mathrm{O}_{4}$ & 9.3 & 0.13 & 0.11 & 9.5 & 0.13 & 0.15 & 6.1 & 0.01 & $2.85 \times 10^{-2}$ & 7.0 & 0.13 & 0.11 \\
\hline $\mathrm{MgSiO}_{3}$ & 84.9 & 0.08 & $6.58 \times 10^{-6}$ & 61.6 & 0.08 & $2.67 \times 10^{-5}$ & 3.0 & 0.11 & $2.68 \times 10^{-3}$ & 61.5 & 0.08 & $2.74 \times 10^{-5}$ \\
\hline $\mathrm{Mg}_{2} \mathrm{SiO}_{4}$ & 68.9 & 0.00 & 0.43 & 50.5 & 0.11 & 0.43 & 4.3 & 0.13 & $8.60 \times 10^{-2}$ & 50.4 & 0.11 & 0.43 \\
\hline $\mathrm{AC}$ & 90.4 & 0.13 & $7.15 \times 10^{-2}$ & 105.2 & 0.13 & 0.12 & 26.0 & 0.14 & 0.11 & 103.2 & 0.13 & 0.12 \\
\hline $\mathrm{SiO}_{2}$ & 55.5 & 0.16 & 0.19 & 38.9 & 0.16 & 0.187 & 4.5 & 0.03 & $1.11 \times 10^{-2}$ & 39.0 & 0.16 & 0.187 \\
\hline$\overline{M_{\mathrm{TOT}}}$ & & & 0.84 & & & 0.92 & & & 0.245 & & & 0.88 \\
\hline
\end{tabular}

mass of the shocked and unshocked ejecta is $\sim 3-4 M_{\odot}$ (Hwang \& Laming 2012), and the mass of gas swept away in the circumstellar medium is $\sim 8 M_{\odot}$ (Hwang \& Laming 2009; Patnaude \& Fesen 2009). Adding the mass of a neutron star remnant, the total mass of the progenitor is estimated to be $\sim(15 \pm 3) M_{\odot}$. The mass of dust associated with the SNR inferred both from Herschel observations and $850 \mu \mathrm{m}$ polarimetry data is $M_{\text {dust }}=0.1-1 M_{\odot}$. Given the large uncertainty in the mass of ${ }^{56} \mathrm{Ni}$, we adopted an intermediate value as input parameter of the simulation.

\subsection{Crab nebula}

The small progenitor mass $\left(8-10 M_{\odot}\right)$, the low explosion en$\operatorname{ergy}\left(10^{50} \mathrm{erg}\right)$ and mass of synthesized ${ }^{56} \mathrm{Ni}\left(0.009-0.016 M_{\odot}\right)$, which are significantly lower than the energy and nickel mass expected for ordinary $\mathrm{SNe}$, all suggest that the Crab could be the result of an electron-capture SN event (Smith 2013). The adopted ${ }^{56} \mathrm{Ni}$ mass for the simulation is intermediate between the observed ones.

\subsection{SNR N49}

The estimated physical properties of N49 have been taken from Park et al. (2012). Otsuka et al. (2010) reported a mass of warm dust of $\sim 0.1-0.4 M_{\odot}$. Emission from a high mass of cold dust is also observed, but it is not possible to separate the contribution coming from dust in the unshocked ejecta from that in the parent cloud.

For this source, no estimate of the ${ }^{56} \mathrm{Ni}$ mass is available. We therefore adopted the same value as we used to model SN 1987A.

\section{Structure of the ejecta and grain distribution}

Starting from a homogeneous set of solar metallicity presupernova models with masses in the range [13-130] $M_{\odot}$ (Chieffi \& Limongi 2013) simulated by means of the FRANEC stellar evolutionary code (Limongi \& Chieffi 2006), we selected the most suitable model for each SN according to the physical properties listed in Table 1. These four selected models were then used as input for the dust formation code (BS07), where classical nucleation theory in steady-state conditions was applied. Here we used the latest version of the dust formation model, which implements an upgraded molecular network, as described in detail in Marassi et al. (2015). We followed the formation of six different dust grain species: amorphous carbon (AC), corundum $\left(\mathrm{Al}_{2} \mathrm{O}_{3}\right)$, magnetite $\left(\mathrm{Fe}_{3} \mathrm{O}_{4}\right)$, enstatite $\left(\mathrm{MgSiO}_{3}\right)$, forsterite $\left(\mathrm{Mg}_{2} \mathrm{SiO}_{4}\right)$, and quartz $\left(\mathrm{SiO}_{2}\right)$.
In Table 2 we show the resulting dust composition and mass distribution. The grains that condense and accrete in SN ejecta follow a log-normal size distribution function of the form

$m_{i}(a)=m_{\max , i} \exp \left[-\frac{\log _{10}^{2}\left(a / a_{0, i}\right)}{2 \sigma_{i}^{2}}\right]$,

where $m_{\max , i}$ represents the distribution maximum, $a_{0, i}$ is the centroid, $\sigma_{i}$ is the width of the distribution, and

$M_{\text {dust }, i}=\int_{0}^{\infty} m_{i}(a) \mathrm{d} a$

is the total dust mass for the $i$ th grain species. Table 2 shows $a_{0}$, $\sigma$ and $M_{\text {dust }}$ for the six dominant grain species in each SN.

In this work we consider a carbon grain density $\rho=$ $2.2 \mathrm{~g} \mathrm{~cm}^{-3}$. However, presolar grain measurements indicate a wide range of density for carbonaceous grains formed in $\mathrm{SNe}$ (e.g. Amari et al. 2005, 2014). We explore this scenario in Appendix A.

\section{GRASH_Rev code}

\subsection{Gas dynamics}

The dynamics of the expansion of a supernova is classically divided into three main stages (see Fig. 1):

1. Ejecta-dominated (ED) expansion phase: as the supernova explodes, a shock wave is triggered and propagates into the ISM (forward shock, FS). At the same time, a reverse shock (RS) propagates into the ejecta. The ejecta is in free expansion.

2. Sedov-Taylor (ST) phase: the mass of the interstellar material swept up by the shock wave becomes comparable to the mass of the ejecta. The ejecta is in adiabatic expansion and the radiative cooling is negligible (Taylor 1950; Sedov 1969).

3. Pressure-driven snowplough (PDS) phase: the shock wave slows down to velocities $\lesssim 200 \mathrm{~km} \mathrm{~s}^{-1}$ and radiative cooling becomes the main cooling process. The outermost shell of the ejecta is pushed into the ambient medium by the pressure of the hot gas inside it.

Finally, when the velocity of the shock front becomes low enough $\left(\sim 10 \mathrm{~km} \mathrm{~s}^{-1}\right)$, the remnant merges with the ISM.

A unified and self-similar solution for the evolution of a SNR from the ED phase through the ST phase was found by Truelove \& McKee (1999) by comparing with the results of a hydrodynamical non-radiative simulation. They considered the following 


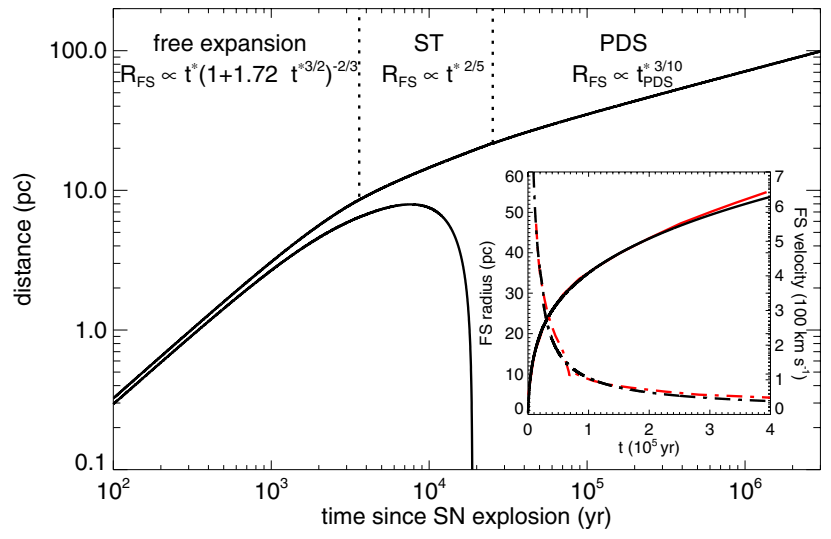

Fig. 1. Position of the forward and reverse shocks (black lines) as a function of time from the ED phase through the PDS phase. In the inset we compare the position (solid line) and velocity (dot-dashed lines) of the forward shock computed using the analytical solution (black lines) with the simulation by Slavin et al. (2015; red lines).

characteristic scales of length and time:

$R_{\mathrm{ch}} \equiv M_{\mathrm{ej}}^{1 / 3} \rho_{0}^{-1 / 3}$,

$t_{\mathrm{ch}} \equiv E_{\mathrm{ex}}^{-1 / 2} M_{\mathrm{ej}}^{5 / 6} \rho_{0}^{-1 / 3}$,

where $M_{\mathrm{ej}}$ is the mass of the ejecta, $\rho_{0}$ is the ISM density, and $E_{\mathrm{ex}}$ is the explosion energy. The position of the forward $\left(R_{\mathrm{FS}}\right)$ and reverse $\left(R_{\mathrm{RS}}\right)$ shocks with respect to the centre of the ejecta for uniform ejecta are well described by

$$
\begin{aligned}
& R_{\mathrm{FS}}\left(t^{*}\right)=2.01 R_{\mathrm{ch}} t^{*}\left(1+1.72 t^{* 3 / 2}\right)^{-2 / 3}, \\
& R_{\mathrm{RS}}\left(t^{*}\right)=1.83 R_{\mathrm{ch}} t^{*}\left(1+3.26 t^{* 3 / 2}\right)^{-2 / 3}
\end{aligned}
$$

during the ED phase, while during the ST phase these two quantities can be approximated by

$$
\begin{aligned}
& R_{\mathrm{FS}}\left(t^{*}\right)=R_{\mathrm{ch}}\left(1.42 t^{*}-0.254\right)^{2 / 5}, \\
& R_{\mathrm{RS}}\left(t^{*}\right)=R_{\mathrm{ch}} t^{*}\left(0.779-0.106 t^{*}-0.533 \ln t^{*}\right),
\end{aligned}
$$

where $t^{*}=t / t_{\text {ch }}$.

When the forward shock reaches velocities $\$ 200 \mathrm{~km} \mathrm{~s}^{-1}$, these equations no longer represent a good approximation, and radiative cooling must be taken into account. Cioffi et al. (1988) found the fiducial time for the onset of the PDS phase to be

$t_{\mathrm{PDS}}=1.33 \times 10^{4} \frac{E_{51}^{3 / 14}}{\zeta_{\mathrm{m}}^{5 / 14} n_{0}^{4 / 7}} \mathrm{yr}$,

where $E_{51}=E_{\mathrm{ex}} / 10^{51} \mathrm{erg}, \zeta_{\mathrm{m}}=1$ for solar abundances and $n_{0}$ is the ISM number density. They also found that the forward shock can be approximated with the following analytical expression:

$R_{\mathrm{FS}}=R_{\mathrm{PDS}}\left(\frac{4}{3} t_{\mathrm{PDS}}^{*}-\frac{1}{3}\right)^{3 / 10}$,

where $t_{\mathrm{PDS}}^{*}=t / t_{\mathrm{PDS}}$ and

$R_{\mathrm{PDS}}=14 \frac{E_{51}^{2 / 7}}{\zeta_{\mathrm{m}}^{1 / 7} n_{0}^{3 / 7}} \mathrm{pc}$

is the position of the forward shock at the time $t_{\mathrm{PDS}}$.
The validity of these analytical solutions is guaranteed by the comparison with hydrodynamical simulations by Cioffi et al. (1988) and Truelove \& McKee (1999). As a further check, we compared the analytical approximations with recent hydrodynamical simulations (Slavin et al. 2015). In the inset in Fig. 1 we show the analytical forward shock position and velocity (black lines) and those retrieved from Fig. 2 in Slavin et al. (2015; red lines). The analytical solution is a good approximation throughout the considered time interval, and the discrepancy in the position of the forward shock is never larger than $\sim 2 \%$.

\subsection{Simulation grid dynamics}

We divided the ejecta into $N_{\mathrm{s}}$ spherical shells and assumed that they all have the same width $\Delta R=R_{\mathrm{ej}} / N_{\mathrm{s}}$, where $R_{\mathrm{ej}}$ is the ejecta radius. The velocity of each shell is initially determined by the homologous expansion of the ejecta:

$v_{j}=v_{\mathrm{ej}} \frac{R_{j}}{R_{\mathrm{ej}}}, \quad v_{\mathrm{ej}}=\sqrt{\frac{10 E_{\mathrm{ex}}}{3 M_{\mathrm{ej}}}}$,

where $v_{j}$ is the velocity of the $j$ th shell and $v_{\mathrm{ej}}$ is the velocity of the ejecta radius. Additional $N_{\mathrm{s}}$ spherical shells are defined to model the ISM around the expanding ejecta. We considered a maximum distance of $R_{\max } \sim 200 \mathrm{pc}$ from the centre of the ejecta and divided the space between $R_{\mathrm{ej}}$ and $R_{\max }$ in $N_{\mathrm{s}}$ shells of equal width $\Delta R_{\mathrm{ISM}}=\left(R_{\max }-R_{\mathrm{ej}}\right) / N_{\mathrm{s}}$. At the beginning of the simulation, these ISM shells are at rest.

We then let the system evolve. The simulation time step, $\Delta t$, is uniquely determined by the dynamics of the reverse shock. It is defined as the time interval during which the reverse shock crosses one shell inside the ejecta, until it reaches the centre of it. At each given time, shells that have not yet been crossed by the reverse shock continue to expand homologously and adiabatically. This implies that the external and internal boundaries of each shell expand at their initial velocity, leading to a larger volume and to a lower density. The gas temperature in the shell changes according to

$T_{j}^{\prime}=T_{j}\left(\frac{V_{j}}{V_{j}^{\prime}}\right)^{1 / \gamma}$,

where $T_{j}$ and $V_{j}$ are the temperature and volume of the $j$ th shell at a given time $t$, the primed variables are the same quantities at time $t+\Delta t$, and $\gamma$ is the adiabatic index (assumed to be $\gamma_{\mathrm{SN}}=1.41$, Chieffi \& Limongi 2013, for the gas in the ejecta and $\gamma_{\mathrm{ISM}}=5 / 3$ for the ISM). Similarly, shells in the ISM that have not yet been crossed by the forward shock remain at rest and their gas properties remain unchanged.

In contrast, when a shell is invested by the reverse or forward shock, it experiences strong variations in density, velocity, and temperature that are well described by the standard RankineHugoniot jump conditions:

$\rho_{j}^{\prime}=\frac{\gamma+1}{\gamma-1} \rho_{j}$,

$v_{j}^{\prime}=v_{j}-\frac{2}{\gamma+1} v_{\mathrm{sh}}$,

$T_{j}^{\prime}=2 \frac{\gamma-1}{(\gamma+1)^{2}} \frac{m}{k_{\mathrm{B}}} v_{\mathrm{sh}}^{2}$

where $\rho_{j}^{\prime}, v_{j}^{\prime}$ and $T_{j}^{\prime}$ are the density, velocity, and temperature of the $j$ th shell (whether in the ejecta or in the ISM) after the passage of the shock, $m$ is the mean particle mass, $k_{\mathrm{B}}$ the Boltzmann 
constant, and $v_{\mathrm{sh}}$ is the velocity of the shock in the reference frame of the shell before it is invested by the shock. This velocity corresponds to $\tilde{v}_{\mathrm{RS}}$ and $v_{\mathrm{FS}}$ for the reverse and forward shocks, whose analytical expressions can be found in Truelove \& McKee (1999).

The velocity of shells between the reverse and forward shocks (hit by a shock at earlier times) is obtained by interpolating between the velocity of the reverse and forward shock. As in Bianchi \& Schneider (2007), we compared the dynamical evolution predicted by the model with the output of numerical simulations by van der Swaluw et al. (2001) and Del Zanna et al. (2003); they agree well until $t \sim 10^{4} \mathrm{yr}$.

The reverse shock velocity in the reference frame of the unshocked ISM reaches an inversion point at $t=t_{\mathrm{rev}}$ and then achieves negative velocities, causing the implosion of part of the ejecta. This process has been investigated in detail through numerical simulations by Cioffi et al. (1988). Before reaching the centre of the ejecta, the temperature and pressure in the core of the ejecta increase, effectively reflecting the reverse shock, and lead to the so-called echoes of thunder described in their work. To qualitatively reproduce this mechanism, we made the following approximation: for all the shells of the ejecta that are invested by the reverse shock after $t_{\text {rev }}\left(j<j^{*}\right.$ with $j^{*}$ the shell invested by the reverse shock at $t_{\text {rev }}$ ), we set the shell velocity to zero, therefore assuming that the bouncing of the reverse shock would effectively cancel out the inward velocity. The velocity of these shells will remain zero until the end of the simulation, and shells in between the $j^{*}$ th shell and the forward shock have velocities obtained from interpolation. During this phase, radiative cooling of the gas by atomic processes starts to be important and is taken into account using the cooling function by Raymond et al. (1976). The error made on the position of the outermost shell of the ejecta computed by this model is always smaller than a factor of $\sim 2$ when compared with the results of numerical simulations (Cioffi et al. 1988). Moreover, since only a quarter of the ejecta is invested by the reverse shock after $t_{\mathrm{rev}}$, all these approximations affect only $\lesssim 2 \%$ of the dust mass.

\subsection{Dust coupling}

The dynamics of dust is governed by its coupling to the gas. Initially, dust is coupled to the unshocked ejecta and uniformly distributed. For a given shell, $j$, both gas and dust have the same velocity, $v_{j}$. The passage of the reverse shock in the $j$ th shell of the ejecta will affect the dynamics of both gas and dust in that shell. The gas is instantaneously modified according to Eq. (11) and takes a velocity $v_{j}^{\prime}$, while dust grains, having more inertia, decouple from the gas and move with an initial velocity (see Eq. (11)) of

$$
\begin{aligned}
\left|v_{\text {rel }}\right| & =\left|v_{j}^{\prime}-v_{j}\right| \\
& =\frac{2}{\gamma+1} v_{\text {sh }}
\end{aligned}
$$

with respect to the shocked gas.

In the model by BS07 dust grains were kept in the same shell for the whole simulation without considering their full dynamics. In addition, once the reverse shock invested a given shell, the grains in that shell were assumed to be at constant velocity (given by Eq. (12)) to take the grain erosion upper limit, without considering the action of drag forces. However, depending on the gas and dust properties, dust grains modify their velocity

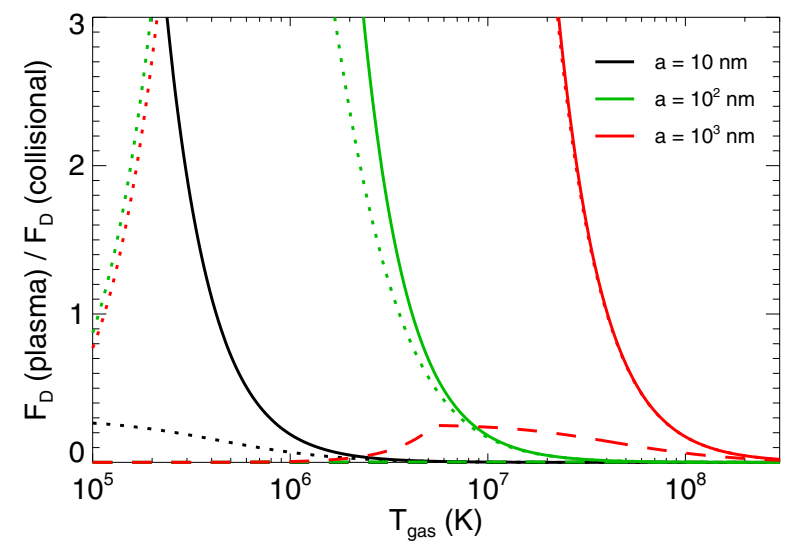

Fig. 2. Ratio between plasma and collisional drag as a function of the gas temperature for different grain radii. Drift velocities are indicated by the line style $\left(V_{\text {rel }}=10,100\right.$, and $1000 \mathrm{~km} \mathrm{~s}^{-1}$ for solid, dotted, and dashed lines, respectively).

according to the following equation (McKee et al. 1987):

$\frac{\mathrm{d} y_{\mathrm{rel}}}{\mathrm{d} t}=\frac{\mathrm{d} \chi}{\mathrm{d} t} \frac{y_{\mathrm{rel}}}{2 \chi}-\frac{F_{\mathrm{D}}}{m_{\mathrm{gr}}}$,

where $\chi$ is the shock compression, $F_{\mathrm{D}}$ the coupling force and $m_{\mathrm{gr}}$ the grain mass. The first term on the right-hand side is known as betatron acceleration (Cowie 1978; Shull 1978). In the presence of a magnetic field, $B$, charged (with charge $|q|$ ), dust grains gyrate around magnetic field lines with a trajectory determined by the Larmor radius:

$r_{\mathrm{L}}=\frac{m_{\mathrm{gr}} v_{\mathrm{rel}, \perp}}{|q| B}$

where $v_{\mathrm{rel}, \perp}$ is the component of the relative velocity between gas and dust which is perpendicular to magnetic field lines. A rapid increase in the gas density due to shock compression will then lead to an enhancement of the magnetic field strength and a consequent (betatron) acceleration and reduction of the Larmor radius. In contrast, the coupling term reduces the grain velocity as a result of the combined effect of collisional (direct) coupling and of plasma drag (Draine \& Salpeter 1979b):

$F_{\mathrm{D}}=F_{\mathrm{D}}($ collisional $)+F_{\mathrm{D}}($ plasma $)$.

The relevant relations that define plasma drag are reported in Eqs. (3)-(6) of Bocchio et al. (2014).

In this model we do not describe the ionisation state of the gas. However, for a sufficiently high gas temperature we can consider the gas to be fully ionised. Therefore the shocked gas is assumed to be composed by $\mathrm{H}^{+}, \mathrm{He}^{++}$and $\mathrm{O}^{+}$, which represent the most abundant species. In Fig. 2 we show the ratio between plasma and collisional drag as a function of the gas temperature and for different grain sizes and drift velocities. Plasma drag has a negligible effect on the dynamics of small grains, while it exceeds collisional drag for $\sim 100 \mathrm{~nm}$ grains at gas temperatures $\lesssim 5 \times 10^{6} \mathrm{~K}$. However, if the velocity is sufficiently high $\left(v_{\text {rel }} \sim 1000 \mathrm{~km} \mathrm{~s}^{-1}\right)$, plasma drag is negligible at all gas temperatures and for all grain sizes.

It has been shown that betatron acceleration efficiently affects the dynamics of interstellar dust grains invested by supernova-triggered shock waves (Jones et al. 1994, 1996; Bocchio et al. 2014). However, this process is probably not relevant for dust produced in SNe. There is evidence for magnetic 
field lines distributed radially inside the ejecta both from polarimetry observations (e.g. Dunne et al. 2009) and from numerical simulations (e.g. Inoue et al. 2013; Schure \& Bell 2014). In the case of symmetric ejecta, the velocity of grains is mostly oriented radially and betatron acceleration is therefore negligible inside the ejecta. In the ISM, shock compression is important during the PDS phase, when the shock front is at low velocities $\left(v_{\mathrm{sh}} \lesssim 200 \mathrm{~km} \mathrm{~s}^{-1}\right)$ and the gas cools as the result of the emission of radiation. However, dust grains ejected from the interior of the $\mathrm{SN}$ are almost at rest with the post-shock gas during this phase (see Sect. 5), and they are therefore not affected by the betatron acceleration. Hence we do not include betatron acceleration in this version of the code.

\subsection{Dust grain number matrix}

to keep track of all the grains in the simulation, we defined a matrix, $\mathfrak{M}$, where - for each of the $2 N_{\mathrm{s}}$ shells and for each relative velocity between grains and gas in the shell - we stored the number of grains together with their size and composition. Initially, the grains occupy the matrix elements corresponding to the shells in the ejecta and zero relative velocity. At each time step, grains are displaced according to the laws described above and the matrix elements are updated. Particular care is required when drag forces are too strong and grain velocities change too quickly during one simulation time step. In that case the time step is divided into $n_{i}=50$ time intervals (each of duration $\delta t$ ), and the grain velocity, $v_{t+\Delta t}$, and position, $d_{t+\Delta t}$, are determined by a sub-grid model following:

$$
\begin{aligned}
& v_{t+\Delta t}=v_{\mathrm{t}}+\sum_{i=1}^{n_{i}}\left(\frac{\mathrm{d} v}{\mathrm{~d} t}\right)_{i} \delta t, \\
& d_{t+\Delta t}=d_{\mathrm{t}}+\sum_{i=1}^{n_{i}} v_{i} \delta t,
\end{aligned}
$$

where $v_{i}$ is the intermediate velocity at each time $t+i \delta t$. Computing the change in grain radius, $a$, at each of the $n_{i}$ time intervals would have been too time-consuming, and we therefore accounted for the grain erosion in this way:

$a_{t+\Delta t}=a_{\mathrm{t}}+\frac{1}{2}\left(\left.\frac{\mathrm{d} a}{\mathrm{~d} t}\right|_{v=v_{\mathrm{t}}}+\left.\frac{\mathrm{d} a}{\mathrm{~d} t}\right|_{v=v_{t+\Delta t}}\right)$

where $\frac{\mathrm{d} a}{\mathrm{~d} t}$ is the grain radius derivative calculated as described in Sect. 4.5 and the subscript indicates the velocity at which the processing is calculated. The grain radius generally does not change very rapidly and a division into sub-grid time intervals is not necessary.

\subsection{Dust processing}

We considered four main physical processes:

1. Sputtering due to the interaction of dust grains with particles in the gas;

2. sublimation due to collisional heating to high temperatures;

3. shattering into smaller grains due to grain-grain collisions; and

4. vapourisation of part of the colliding grains during graingrain collisions.

The sputtering process can be estimated following the formalism by Tielens et al. (1994) as revisited by Bocchio et al. (2014). The
Table 3. Parameters for the analytical modelling of the size-dependent sputtering.

\begin{tabular}{ccccccc}
\hline \hline & $\mathrm{Al}_{2} \mathrm{O}_{3}$ & $\mathrm{Fe}_{3} \mathrm{O}_{4}$ & $\mathrm{MgSiO}_{3}$ & $\mathrm{Mg}_{2} \mathrm{SiO}_{4}$ & $\mathrm{AC}$ & $\mathrm{SiO}_{2}$ \\
\hline $\mathrm{p}_{1}$ & 1.1 & 1.2 & 1.0 & 1.5 & 4.9 & 0.85 \\
$\mathrm{p}_{2}$ & 0.39 & 0.55 & 0.50 & 1.2 & 0.55 & 1.2 \\
$\mathrm{p}_{3}$ & 1.1 & 1.0 & 1.0 & 0.57 & 0.77 & 0.56 \\
$\mathrm{p}_{4}$ & 1.9 & 1.9 & 1.8 & 1.1 & 4.7 & 1.1 \\
$\mathrm{p}_{5}$ & 2.1 & 2.0 & 2.1 & 0.52 & 3.0 & 1.6 \\
$\mathrm{p}_{6}$ & 0.78 & 0.80 & 0.76 & 0.37 & 1.2 & 0.29 \\
\hline
\end{tabular}

sputtering rate is expressed as

$\frac{\mathrm{d} N_{\mathrm{sp}}}{\mathrm{d} t}=2 \pi a^{2} \sum_{i} n_{i}\left\langle Y_{i} v\right\rangle$,

where $\frac{\mathrm{d} N_{\mathrm{sp}}}{\mathrm{d} t}$ is the number of sputtered atoms per unit time, $a$ is the grain radius, $n_{i}$ the number density of the $i$ th ion and

$\left\langle Y_{i} v\right\rangle=\int Y_{i} v f_{\mathrm{skM}}(v) \mathrm{d} v$,

where $Y_{i}$ is the sputtering yield (assuming a semi-infinite target) of the $i$ th ion, $v$ is the relative velocity between grain and gas particle (a combination of the thermal and drift velocities), and $f_{\text {skM }}(v)$ is the skewed Maxwellian distribution (Shull 1978; Guillet 2008; Bocchio et al. 2014). We used the same sputtering yields as derived by Tielens et al. (1994) and used by BS07 and Nozawa et al. (2006, 2007).

Energetic ions can have a penetration depth that is comparable or even larger than the smallest grains considered in this work. For example, a $\sim 1 \mathrm{keV} \mathrm{He}{ }^{+}$ion (corresponding to the energy of an ion in a hot gas with $T_{\mathrm{g}} \sim 8 \times 10^{6} \mathrm{~K}$ ) has a penetration depth $R_{\mathrm{p}} \sim 10 \mathrm{~nm}$ in graphite and therefore it is likely to cross grains of radius smaller than $R_{\mathrm{p}}$ without leading to any sputtering of the target. This effect was taken into account in models by Jurac et al. (1998) and Serra Díaz-Cano \& Jones (2008) and then adopted by Bocchio et al. (2014) for sputtering of interstellar carbonaceous grains in supernova-triggered shocks. Using a similar approach, here we extend the latter study to simulate this effect for all the target materials considered in GRASH_Rev. The sputtering yield, $Y_{\mathrm{a}}$, of a grain of radius $a$ is then expressed as

$Y_{\mathrm{a}}=Y_{\infty} f(x)$,

where $Y_{\infty}$ is the sputtering yield for a semi-infinite target, $x=$ $a /\left(0.7 R_{\mathrm{p}}\right)$ and $f(x)$ is a function that can be parametrised as

$f(x)=1+p_{1} \exp \left[-\frac{\left(\ln x / p_{2}\right)^{2}}{2 p_{3}^{2}}\right]-p_{4} \exp \left[-\left(p_{5} x-p_{6}\right)^{2}\right]$.

The function $f(x)$ depends on the target material, and the six parameters we used are reported in Table 3. In general, this function tends to unity for sufficiently large grains (with respect to $R_{\mathrm{p}}$, i.e. $x \rightarrow \infty$ ) and tends to zero for small grains (i.e. $x \rightarrow 0$ ). For grain radii comparable to the ion penetration depth, the sputtering yield is enhanced and can reach values $Y_{\mathrm{a}} \sim 4 Y_{\infty}$ for graphitic-type grains. For a detailed explanation of the method used to retrieve the parameters involved in Eq. (21), we refer to Appendix B. Finally, since the mass of (sub-)nanoparticles is negligible for all the SN models considered, we did not implement any molecule dissociation process (e.g. Micelotta et al. 2010b,a) as was done in Bocchio et al. (2014). 
Sublimation is implemented with the same method as described by BS07, using the thermal sublimation rates by Guhathakurta \& Draine (1989) and Draine \& Hao (2002). In agreement with BS07, we find this process to provide a negligible contribution to dust destruction for all the SN models.

Grain-grain collisions lead to the formation of craters in the two colliding grains. The matter ejected from the crater is fragmented or even vapourised and brought back to the gas phase. Shattering and vapourisation processes were implemented following equations by Jones et al. (1994, 1996). However, some of the grain materials present in this work were not in the original papers. We adopted values from the literature for the different grain properties (see Table B.1). For a detailed description of the computation of the relevant quantities we refer to Appendix C.

Given the low density of dust in the ejecta, grain-grain collisions are rare, and shattering and vapourisation lead to minor processing with respect to sputtering. This result can be better understood if we calculate the timescale between grain-grain collisions, $\Delta t_{\text {coll }}$. Particles with a mean number density $\bar{n}$ and a typical radius $a$, in motion in a given volume will have a mean free path:

$\lambda=\frac{1}{\pi a^{2} \bar{n}}$,

and the timescale between two collisions is given by

$\Delta t_{\mathrm{coll}}=\frac{\lambda}{\bar{v}}$,

with $\bar{v}$ the typical relative velocity between particles. As an example we considered a $S N \sim 10^{4} \mathrm{yr}$ after the explosion. The volume occupied by dust is roughly a sphere of radius $10 \mathrm{pc}$. Assuming $0.5 M_{\odot}$ of silicate grains with an average radius of $50 \mathrm{~nm}$, the mean grain number density is $\bar{n} \sim 10^{-11} \mathrm{~cm}^{-3}$. Then, considering a mean relative velocity of $\bar{v} \sim 500 \mathrm{~km} \mathrm{~s}^{-1}$, the timescale between grain-grain collisions is $\Delta t_{\text {coll }} \sim 8 \times 10^{5} \mathrm{yr}$, showing that these events are rare. Furthermore, with the expansion of the ejecta and the dispersion of grains into the ISM, the frequency of collisions is even lower.

At each time step in the simulation, the grains in all the cells of the matrix $\mathfrak{M}$ are processed by the four mechanisms described above and the matrix is updated.

\section{Results}

We ran the GRASH_Rev code for four SNe with the following input parameters: the number density of the surrounding ISM, the explosion energy, the progenitor mass $\left(n_{0}, E_{\mathrm{exp}}^{\mathrm{mod}}, M_{\mathrm{prog}}^{\mathrm{mod}}\right.$ listed in Table 1), and the initial dust mass, composition, and size distribution ( $M_{\text {dust }}, a_{0}, \sigma$ for each grain species listed in Table 2). The number of shells used for the simulation grid was fixed and equal to $2 N_{\mathrm{s}}=800$. However, we note that this represents a robust parameter; halving or doubling the number of shells, the simulation output remains unchanged.

In the following sections we present the results of the simulations.

\subsection{Test particle dynamics and processing}

To better understand the behaviour of individual dust grains, we first illustrate the dynamics of a few test particles, giving as an input to the code their initial position, size, and material.

We considered the simulation of SNR N49 and defined six test particles made of $\mathrm{Mg}_{2} \mathrm{SiO}_{4}$ with radii of 10,100 ,

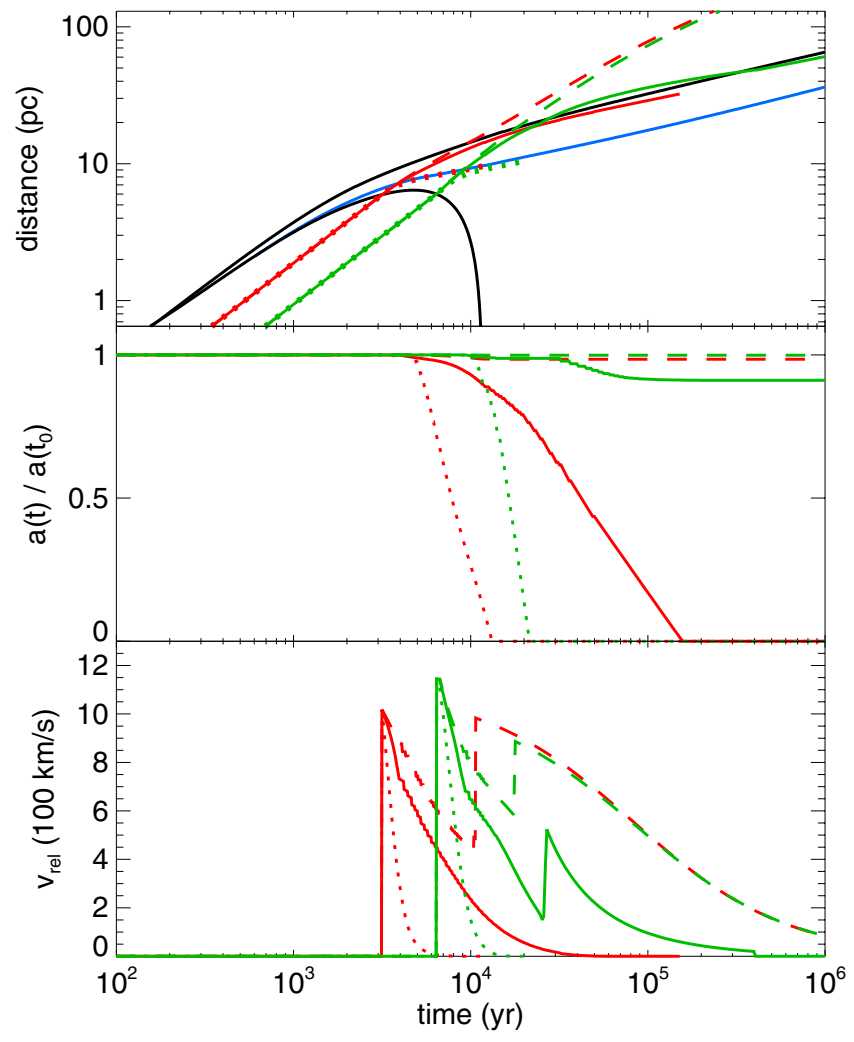

Fig. 3. $\mathrm{Mg}_{2} \mathrm{SiO}_{4}$ test particle trajectory, size, and velocity in the ejecta of SNR N49. Green and red lines indicate particles initially placed at one-forth and half of the radius of the ejecta, respectively. The initial size of particles is 10,100 , and $1000 \mathrm{~nm}$ for dotted, solid and dashed lines, respectively. In the top panel, black lines indicate the positions of forward and reverse shock, and the blue line is the boundary between the ejecta and the ISM.

and $1000 \mathrm{~nm}$ initially placed at a distance of one-forth and half of the initial radius of the ejecta. In Fig. 3 we show their trajectories (upper panel), the size evolution (central panel), and relative velocities with respect to the gas. The positions of forward and reverse shocks are indicated by black lines, while the position of the boundary between the ejecta and the ISM are plotted by the blue line.

Initially all the particles are at rest with respect to the ejecta. When the reverse shock reaches the shell where the particles reside, the gas undergoes a jump in conditions (Eq. (11)), while dust grains are not instantaneously coupled and assume a velocity given by Eq. (12) with respect to the gas. Immediately after the passage of the reverse shock, depending on the gas conditions and grain size, dust grains are slowed by drag forces and processed by collisions with gas particles and other grains.

Small grains ( $a=10 \mathrm{~nm}$, dotted lines) are quickly stopped and destroyed within the ejecta, while larger grains $(a=$ $100,1000 \mathrm{~nm}$, solid and dashed lines, respectively) are eroded to a lower extent. Furthermore, $100 \mathrm{~nm}$ grains that were initially placed at one-forth of the ejecta (green lines) have enough inertia to cross the forward shock, while $100 \mathrm{~nm}$ grains initially placed at half of the ejecta (red lines) are stopped and destroyed in $\sim 10^{5} \mathrm{yr}$.

As soon as a grain crosses the forward shock, its relative velocity with respect to the unshocked ISM gas, $v_{\text {ISM }}^{\prime}$, is enhanced to

$v_{\mathrm{ISM}}^{\prime}=v_{\mathrm{ISM}}+v_{\mathrm{Sh}}$, 


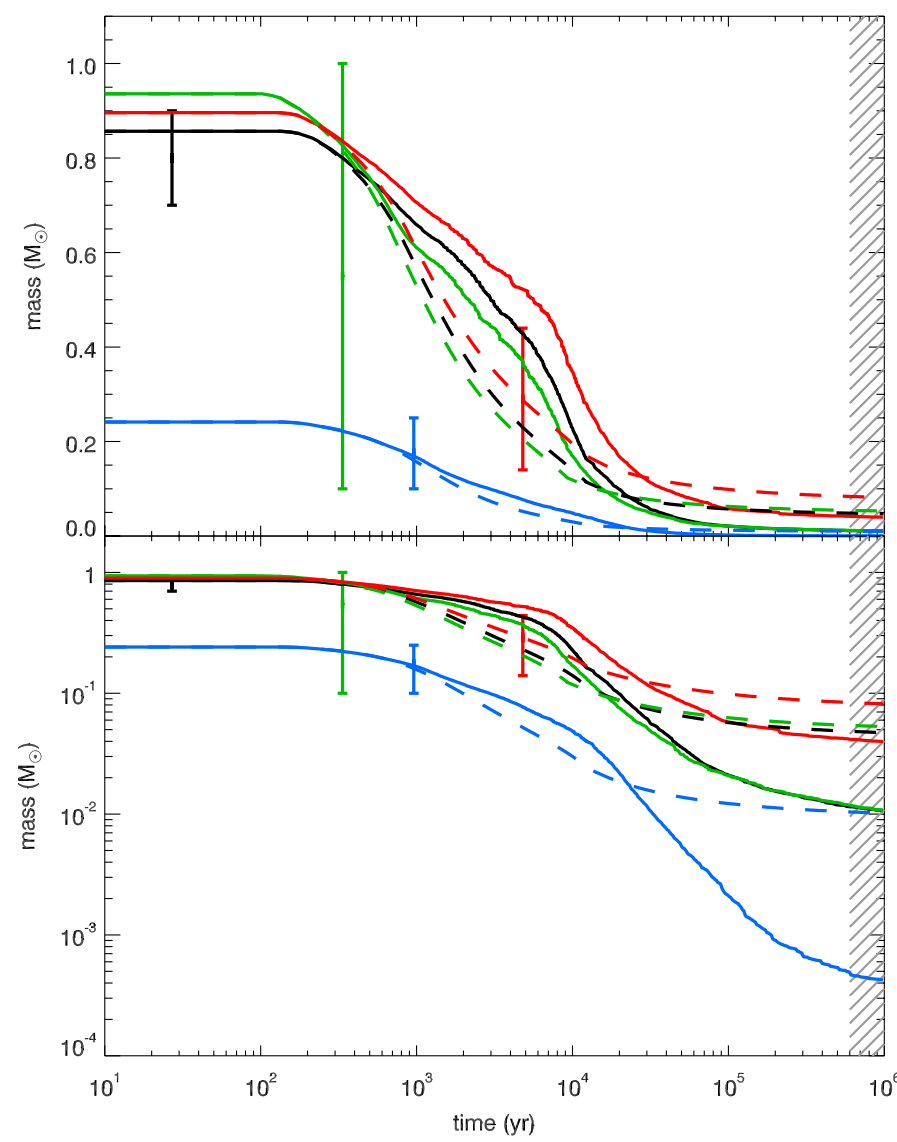

Fig. 4. Dust mass evolution as a function of time for the four $\mathrm{SNe}$ considered in this study (1987A: black, CasA: green, Crab: blue, and N49: red). Solid lines represent GRASH_Rev results and dashed lines the results obtained by BS07 model using the same initial conditions. Data points represent the observed dust masses, and the shaded region indicate the time interval when dust processing fades out.

where $v_{\text {ISM }}$ is the relative velocity between the grain and the shocked ISM and $v_{\text {sh }}$ is the shock velocity. Because of the effect of drag forces, grains will again slow down in the unshocked ISM. If the velocity of a grain is too low in this medium (e.g. green solid line in Fig. 3), it can be reached by the faster forward shock and will cross the shock front a second time. This time, the relative velocity between grain and gas is reduced and the grain is almost at rest in the shocked gas, therefore avoiding further processing.

Hence, it is clear that the fate of individual grains depends not only on its initial size but also on its initial position within the ejecta.

\subsection{Dust mass evolution}

In Fig. 4 we show the time evolution of the dust mass following the SN explosion. The evolution starts at the end of the dust nucleation phase. Each line represents the evolution of a single SN (1987A: black, CasA: green, Crab: blue, and N49: red), and the data points illustrate the dust mass estimated from the observations (see Sect. 2). As a comparison we also show the mass evolution computed using the model by BS07, assuming no drag forces (dashed lines). In the inset we highlight results for the time interval $t=10^{5}-10^{6} \mathrm{yr}$ on a logarithmic scale.

The dust mass estimated using GRASH_Rev simulations agree well with the observations, except for SNR N49, for which

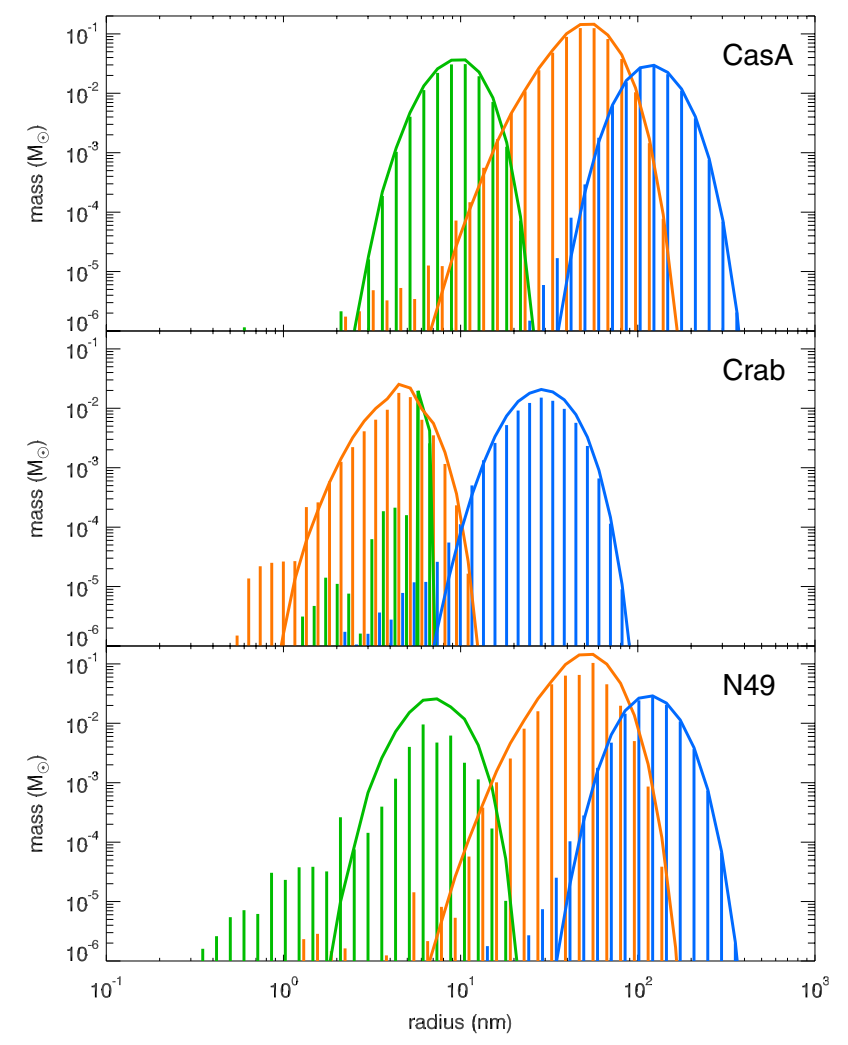

Fig. 5. Dust mass distributions for CasA, Crab, and N49 at formation (solid lines) and observation times (histograms). In each panel we show the three dominant dust species: $\mathrm{Fe}_{3} \mathrm{O}_{4}$ (green), $\mathrm{Mg}_{2} \mathrm{SiO}_{4}$, and $\mathrm{SiO}_{2}$ (summed together in orange) and $\mathrm{AC}$ (blue).

the dust mass predicted by GRASH_Rev is $\sim 15 \%$ higher than the upper mass limit of the range of values inferred from observations $\left(0.4 M_{\odot}\right.$, see Table 1 and Sect. 5.3). A more detailed comparison between model predictions and observations is given in Sect. 5.3.

At times $t \sim 10^{3}-10^{4} \mathrm{yr}$ the reverse shock destruction predicted by GRASH_Rev is less important than that predicted by BS07. Because of the passage of the reverse shock, dust is decoupled from the gas during this time interval and travels in between the reverse and forward shocks where the gas conditions are milder. For $t \gtrsim 10^{4} \mathrm{yr}$, most of the grains reach regions behind the forward shock where gas conditions are harsh and dust erosion is efficient. For all the four $\mathrm{SNe}$, we predict final dust masses that are $\sim 5-50 \%$ of those estimated using the model by BS07, where the displacement of grains and the effect of the forward shock were neglected.

\subsection{Dust mass distribution}

In Fig. 5 we show the predicted mass in different dust species as a function of the grain size for CasA, Crab, and N49. The initial mass distributions (obtained as described in Sect. 3) are shown with solid lines, while the dust mass distributions at the time corresponding to the age of these $\mathrm{SNe}, t_{\mathrm{obs}}$, is represented by the histograms. Only the most abundant species are displayed here: $\mathrm{Fe}_{3} \mathrm{O}_{4}, \mathrm{Mg}_{2} \mathrm{SiO}_{4}$, and $\mathrm{SiO}_{2}$ and $\mathrm{AC}$. We do not show the results for SN 1987A because the reverse shock has not yet affected the mass of dust formed in this SN (see Fig. 4).

The dust mass distribution at the observation time is shifted towards small grains and reduced in mass as a result of dust processing (compare the entries in Tables 2 and 4). 
M. Bocchio et al.: Dust grains from the heart of supernovae

Table 4. Dust masses (expressed in $M_{\odot}$ ) at the observation time, $t_{\mathrm{obs}}$, and at the end of the simulation, $t_{\mathrm{end}}$.

\begin{tabular}{|c|c|c|c|c|c|c|c|c|}
\hline & \multicolumn{2}{|c|}{ 1987A } & \multicolumn{2}{|c|}{ CasA } & \multicolumn{2}{|c|}{ Crab } & \multicolumn{2}{|c|}{ N49 } \\
\hline & $t_{\mathrm{obs}}$ & $t_{\text {end }}$ & $t_{\mathrm{obs}}$ & $t_{\text {end }}$ & $t_{\mathrm{obs}}$ & $t_{\text {end }}$ & $t_{\mathrm{obs}}$ & $t_{\text {end }}$ \\
\hline$M_{\mathrm{Al}_{2} \mathrm{O}_{3}}$ & $2.57 \times 10^{-2}$ & $1.24 \times 10^{-6}$ & $2.14 \times 10^{-2}$ & $3.00 \times 10^{-6}$ & $3.66 \times 10^{-3}$ & $1.51 \times 10^{-6}$ & $6.19 \times 10^{-3}$ & $3.93 \times 10^{-6}$ \\
\hline$M_{\mathrm{Fe}_{3} \mathrm{O}_{4}}$ & 0.11 & $1.46 \times 10^{-5}$ & 0.13 & $6.78 \times 10^{-6}$ & $1.58 \times 10^{-2}$ & $2.00 \times 10^{-6}$ & $3.02 \times 10^{-2}$ & $2.27 \times 10^{-5}$ \\
\hline$M_{\mathrm{MgSiO}_{3}}$ & $6.58 \times 10^{-6}$ & $5.91 \times 10^{-8}$ & $2.4 \times 10^{-5}$ & $1.65 \times 10^{-9}$ & $1.80 \times 10^{-3}$ & $1.87 \times 10^{-6}$ & $1.84 \times 10^{-5}$ & $1.70 \times 10^{-7}$ \\
\hline$M_{\mathrm{Mg}_{2} \mathrm{SiO}_{4}}$ & 0.44 & $2.10 \times 10^{-3}$ & 0.40 & $1.34 \times 10^{-5}$ & $6.19 \times 10^{-2}$ & $1.38 \times 10^{-4}$ & 0.28 & $1.48 \times 10^{-3}$ \\
\hline$M_{\mathrm{AC}}$ & $7.15 \times 10^{-2}$ & $8.50 \times 10^{-3}$ & 0.12 & $1.07 \times 10^{-2}$ & $7.85 \times 10^{-2}$ & $2.57 \times 10^{-4}$ & 0.11 & $3.86 \times 10^{-2}$ \\
\hline$M_{\mathrm{SiO}_{2}}$ & 0.19 & $1.26 \times 10^{-4}$ & 0.16 & $6.15 \times 10^{-6}$ & $6.80 \times 10^{-3}$ & $2.08 \times 10^{-5}$ & $9.65 \times 10^{-2}$ & $3.51 \times 10^{-4}$ \\
\hline$M_{\mathrm{TOT}}$ & 0.84 & $1.07 \times 10^{-2}$ & 0.83 & $1.07 \times 10^{-2}$ & 0.17 & $4.21 \times 10^{-4}$ & 0.52 & $4.02 \times 10^{-2}$ \\
\hline
\end{tabular}

This effect is more evident for the oldest SN remnant, N49. Furthermore, small grains are more affected by processing than large grains, reflecting the behaviour of test particles seen in Fig. 3.

In what follows, the dust size and composition predicted by GRASH_Rev for these three SNe at their respective ages is compared with the dust properties adopted to estimate the dust mass from IR and submm observations.

For CasA our model predicts little destruction, and the three dominant species are characterized by a mass distribution that is similar to the initial one. About $0.77 M_{\odot}$ of dust is still unshocked, and therefore only $0.06 M_{\odot}$ of dust is likely to be warm and collisionally heated by the hot post-shock gas. These values agree well with the mass of cold and warm dust obtained from fitting observations $\left(0.1-1 M_{\odot}\right.$ from Herschel and polarimetry observations and 0.02-0.054 $M_{\odot}$ from Spitzer data, Rho et al. 2008; Arendt et al. 2014).

The Crab nebula has been observed with Herschel at wavelenghts between 51 and $670 \mu \mathrm{m}$ by Gomez et al. (2012). They fit the observed emission with two-temperature blackbodies, inferring a dust mass of $0.11(0.24) M_{\odot}$ when carbonaceous (silicate) grain optical properties are used to fit the data. At the time corresponding to the age of this $\mathrm{SN}$, we predict a total dust mass of $0.17 M_{\odot}$, in good agreement with the observations, with an almost equal contribution from silicates $\left(\sim 0.09 M_{\odot}\right)$ and carbon grains $\left(\sim 0.08 M_{\odot}\right)$. However, the predicted grain composition is more complex than assumed to fit the data. The simulation predicts that $\sim 0.023 M_{\odot}$ of dust lies between the forward and reverse shock, probably emitting at high temperatures, while the warm dust mass inferred from the data is estimated to be 6-8 $\times 10^{-3} M_{\odot}$. However, to fit the data, all grains were assumed to be either at high $\left(T_{\mathrm{w}} \sim 55-63 \mathrm{~K}\right)$ or low temperature $\left(T_{\mathrm{c}} \sim 28-33 \mathrm{~K}\right)$. Given the wide range of predicted grain sizes, this assumption is likely to be a very poor approximation.

Observations of warm dust emission in N49 by Otsuka et al. (2010) indicate a dust mass of $0.1(0.4) M_{\odot}$ if carbonaceous ( silicate) grains are assumed as the main constituent of dust. The cold component is hard to detect observationally as it is strongly contaminated by the emission of dust in the parent cloud at a similar temperature. Only a single dust temperature has been used to fit the data (Otsuka et al. 2010), and this could lead to a large uncertainty in the estimated dust mass (Mattsson et al. 2015). The GRASH_Rev simulation predicts that in SNR N49 the reverse shock has already processed much of the ejecta and only $0.04 M_{\odot}$ of dust is still in the unshocked region, emitting as cold dust. Hence, we predict a warm dust mass of $\sim 0.48 M_{\odot}$, dominated by silicate grains, which is only $\sim 15 \%$ higher than the observed warm dust mass when silicate optical properties are assumed.
Given the uncertainties in inferring the dust mass from the data, we can conclude that the model predictions provide a reasonable estimate of the mass of dust that has survived the passage of the reverse shock. Based on our simulation, we can conclude that a dust mass of [0.7-0.9] $M_{\odot}$, such as the one observed in SN 1987A, can be safely considered to be representative of the efficiency of dust production in massive stars, as the ejecta have not yet been invested by the reverse shock. This conclusion does not apply to the other three $\mathrm{SNe}$ that we have considered, where - at the estimated time from the explosions - between 10 and $40 \%$ of the freshly formed dust has already been destroyed. However, for none of the SNe considered, the reverse shock travelled to the centre of the ejecta. This implies that current observations cannot be used to estimate the mass of dust that will be injected in the interstellar medium, the so-called effective dust yield.

\subsection{Effective $S N$ dust yields}

The final time of the simulation was assumed to be $t_{\mathrm{end}}=10^{6} \mathrm{yr}$ where the velocity of the forward shock is $v_{\text {sh }} \lesssim 10 \mathrm{~km} \mathrm{~s}^{-1}$ and dust grains are either destroyed or embedded in the ISM gas. Table 4 illustrates the predicted dust mass at the end of the simulation. We find that the effective dust masses injected in the ISM from these $\mathrm{SNe}$ will be significantly lower than inferred from present observations: $\sim 1 \%$ of the currently detected mass for SN 1987A and CasA, 8\% for N49 and less than $1 \%$ for the Crab nebula. The resulting dust yields depend on the progenitor mass, explosion energy, and the age of the $\mathrm{SN}$, and lie in the range $\left[4.21 \times 10^{-4}-4.02 \times 10^{-2}\right] M_{\odot}$. Grains in the Crab nebula suffer the largest destruction. The Crab nebula has a lower explosion energy and progenitor mass than the other SNe. This leads to the formation of smaller dust grains (e.g. AC grains have an average size of $a_{0}=26 \mathrm{~nm}$, in the other three SNe $a_{0} \sim 90-100 \mathrm{~nm}$ ) and to a lower dust mass. For the same reason, silicate grains are destroyed more efficiently. As a result, more than $60 \%$ of the dust mass that will be injected in the ISM is in the form of carbonaceous grains. These grains, being larger than grains of other species, have more inertia and, depending on their initial position, can cross both the reverse and forward shock, reaching the unshocked ISM and surviving the explosion.

\section{Discussion and astrophysical implications}

The dust mass obtained from fitting observations was determined under several assumptions that make the measurement rather uncertain.

1. Often, a single dust material and size is used to reproduce the dust emission. However, dust in SNRs is rich in 
composition, and each material has a wide size distribution. This is reflected in the dust temperature distribution: for example small grains are heated to higher temperatures than larger grains, and their emission per unit mass will be higher. Mattsson et al. (2015) noted that depending on the grain size/temperature distribution, the measured dust mass can vary by up to six times the original value, but that most of the complication derives from the assumed grain material.

2. In many cases and especially in young SNe, most of the dust mass resides in the unshocked ejecta and is therefore likely to be cold with a similar temperature to that of dust in the parent cloud (radiative transfer calculations in SN 1987A predict temperatures $T_{\text {dust }}=15-20 \mathrm{~K}$; Wesson et al. 2015). The emissions from these two sources come from the same spatial region and are therefore entangled, and further multiwavelength observations are therefore needed for a correct dust mass evaluation.

3. For CasA and the Crab nebula, recent observations suggest that the unshocked ejecta have a clumpy density distribution (Lee et al. 2015; Owen \& Barlow 2015). When this is taken into account, the inferred dust masses are generally higher. In dust formation and destruction models, a clumpy ejecta would also favour a higher dust mass as (i) the formation of dust grains would be more efficient, therefore creating larger grains; and (ii) the higher contrast in density between the diffuse and clumpy gas would slow the reverse shock, leading to less dust processing.

In addition, the mass of the progenitor stars and the explosion energy for some $\mathrm{SNe}$ are also difficult to reconstruct from the available data. These do not affect the estimated mass of freshly formed dust ${ }^{1}$, but they may affect the destruction efficiencies by the reverse shock. As an example, assuming an increase in the explosion energy by a factor 2 for CasA leads to a $60 \%$ lower dust mass at $t_{\mathrm{obs}}$ than the value reported in Table 4. However, as is clear from Fig. 4, this model would still agree with the observed dust mass, given the large error bars. Hence, the uncertainties that affect the measured dust mass encompass the range of dust masses predicted by the model for different initial explosion parameters.

Given these uncertainties, the models described in this paper appear to reproduce the dust masses in the four $\mathrm{SNe}$ well. The average effective dust yield is estimated to be $(1.55 \pm 1.48) \times$ $10^{-2} M_{\odot}$. This agrees well with the dust yield used in chemical evolution models based on BS07 dust yields with moderate destruction by the reverse shock. For a Salpeter initial mass function (IMF), the average dust mass injected by SNe in the ISM is $(2.50-2.73) \times 10^{-2} M_{\odot}$ for SN progenitors with metallicities $0.1 Z_{\odot} \leq Z \leq 1 Z_{\odot}$ and a circumstellar medium density of $1 \mathrm{~cm}^{-3}$ (Valiante et al. 2009, 2011, 2014; see also Fig. 6 in Schneider et al. 2014).

In the Milky Way, the timescale between the explosion of two SNe has been estimated to be $\sim 40 \mathrm{yr}$ ( $\mathrm{Li}$ et al. 2011). Using the mean effective dust yield that we have obtained leads to a SN dust production rate of $(3.9 \pm 3.7) \times 10^{-4} M_{\odot} \mathrm{yr}^{-1}$. This value can be compared to the dust production rate by AGB stars. Because of source confusion in the Galactic plane, surveys have targeted

\footnotetext{
As an example, changing the explosion energy adopted for CasA from $1.5 \times 10^{51} \mathrm{erg}$ to $3 \times 10^{51} \mathrm{erg}$ induces only a $5 \%$ difference in the dust mass. A similar difference is found if we increase the $\mathrm{Ni}^{56}$ mass adopted for the $\mathrm{Crab}$ from $0.014 M_{\odot}$ to $0.058 M_{\odot}$.
}

the Magellanic Clouds to make a global census of dusty AGB stars, inferring dust production rates of $\sim 2 \times 10^{-5} M_{\odot} / \mathrm{yr}$ for the LMC, Riebel et al. (2012) and of $\sim 8 \times 10^{-7} M_{\odot} / \mathrm{yr}$ for the Small Magellanic Cloud (Boyer et al. 2012), with variations among the results of different surveys (Matsuura et al. 2013), and in good agreement with theoretical predictions (Ventura et al. 2012b,a, 2014). Hence, even in the LMC, the current dust production rate by AGB stars appears to be more than one order of magnitude lower than that estimated from our sample of four SNe. But the relative importance of AGB stars and $\mathrm{SNe}$ as dust producers depends on the initial mass function and on the star formation history of each galaxy (Valiante et al. 2009).

The importance of stellar sources of dust is limited by grain destruction in SN-driven interstellar shocks, however. The dust destruction timescale in the Milky Way has been estimated to be $\tau_{\text {des,Mw }} \sim 10^{8}, 10^{9}$ yr by Bocchio et al. (2014) and Slavin et al. (2015), respectively. Rescaling these values by the ratio between the ISM mass in the Milky Way and in the LMC $\left(M_{\text {ISM,Mw }} / M_{\text {ISM,LMC }} \approx 100\right)$, the dust lifetime in the LMC can be estimated as $\sim 10^{6}-10^{7} \mathrm{yr}$, in agreement with the recent observational study by Lakićević et al. (2015), which leads to $\tau_{\text {des,LMC }} \sim 2 \times 10^{7} \mathrm{yr}$. Then, assuming a total interstellar dust mass in the LMC of $M_{\text {dust }}=7.3 \times 10^{5} M_{\odot}$ (Gordon et al. 2014), the resulting dust destruction rate is $\mathrm{d} M_{\mathrm{des}, \mathrm{LMC}} / \mathrm{d} t \sim$ $3 \times 10^{-2}-7 \times 10^{-1} M_{\odot} \mathrm{yr}^{-1}$. These values are higher than the inferred dust production rates for $\mathrm{SNe}$ and $\mathrm{AGB}$ stars by a factor of $>100$, therefore requiring dust accretion in the gas phase of the LMC. Even if the dust formation timescale in AGB stars and SNe appears to be longer than the destruction timescale, stellar dust production is nevertheless an important mechanism to provide the seeds for grain growth through gas accretion in denser regions (see however Jones \& Nuth 2011).

The result of our study suggests that of all the different dust species that are formed in the ejecta of $\mathrm{SNe}$, carbonaceous grains suffer the least destruction by the reverse shock, which means that they provide the dominant contribution to the effective $\mathrm{SN}$ dust yields. This implies that carbon dust may be present even in the ISM of young galaxies, with relatively unevolved stellar populations. This selection effect is even more pronounced if carbon grains are preferentially formed with densities lower than graphite $\left(\rho<2.2 \mathrm{~g} \mathrm{~cm}^{-3}\right.$, see Appendix A).

Finally, it is not straightforward to use the results of this study as a way to estimate the mass of dust produced by SNe in galaxies at high redshifts. Although very little is known about their ISM conditions, if these SNe explode in a circumstellar medium of larger density, they experience a harder reverse shock and presumably a stronger dust destruction. As an example, if exploded in a circumstellar medium density of $n_{0} \sim 15 \mathrm{~cm}^{-3}-$ a factor 10 larger than assumed here - the same simulation used to describe SN 1987A predicts a final dust mass of $3.4 \times 10^{-4} M_{\odot}$, which is $\sim 30$ lower than our standard run.

\section{Conclusions}

We have presented GRASH_Rev, a new code that describes dust processing in a SN explosion, improving the existing BS07 model by treating the full dynamics of dust grains within the ejecta and in the surrounding ISM. We applied the model to four well-studied SNe in our Galaxy and in the LMC: SN 1987A, CasA, the Crab nebula, and N49. Multi-wavelength observations with Spitzer and Herschel allow inferring the mass of warm and cold dust associated with the ejecta. 
We find that our models are able to well reproduce the observed dust mass and can therefore be used to estimate the effective dust yield, hence the mass of dust produced by $\mathrm{SNe}$ that is able to enrich the ISM.

For SN 1987A, the ejecta have not yet been reprocessed by the reverse shock. Hence, the currently observed dust mass of [0.7-0.9] $M_{\odot}$ is indicative of the efficiency of dust formation in SN ejecta. Conversely, between 10 and $40 \%$ of the newly formed dust has already been destroyed in the other three SNe. The simulations show that sputtering due to the interaction of dust grains with particles in the gas represents the dominant destruction process because physical conditions in the ejecta cause sublimation to be almost negligible and grain-grain collisions to be extremely rare.

Since the largest dust destruction occurs between $10^{3}$ and $10^{5} \mathrm{yr}$ after the explosion, current observations that sample relatively young $\mathrm{SNe}$ (with age $<4800 \mathrm{yr}$ ) only provide an upper limit on the effective dust yield. Our models indicate that only between 1 and $8 \%$ of the currently observed dust mass will contribute to the enrichment of the ISM, with an average SN effective dust yield of $(1.55 \pm 1.48) \times 10^{-2} M_{\odot}$, largely dominated by carbonaceous grains. This agrees well with the IMFaveraged yields adopted in chemical evolution models (Valiante et al. 2009, 2011, 2014; de Bennassuti et al. 2014; Schneider et al. 2014) that account for SN reverse-shock destruction. When compared to dust destruction efficiencies in SN-driven interstellar shocks that were recently estimated by theoretical models (Bocchio et al. 2014; Slavin et al. 2015) and observations (Lakićević et al. 2015), these figures imply that SNe may be net dust destroyers, pointing to grain growth in the ISM as the dominant dust enrichment process both in local galaxies and at high redshifts (Valiante et al. 2014; de Bennassuti et al. 2014; Michałowski 2015; Mancini et al. 2015).

Acknowledgements. The research leading to these results has received funding from the European Research Council under the European Union (FP/20072013) / ERC Grant Agreement n. 306476.

\section{References}

Amari, S., Zinner, E., \& Lewis, R. S. 2005, in 36th Annual Lunar and Planetary Science Conf., eds. S. Mackwell, \& E. Stansbery, Lunar and Planetary Inst. Tech. Rep., 36, 1867

Amari, S., Zinner, E., \& Gallino, R. 2014, eds. S. Jeong, N. Imai, H. Miyatake, \& T. Kajino, AIP Conf. Ser., 1594, 307

Arendt, R. G., Dwek, E., Kober, G., Rho, J., \& Hwang, U. 2014, ApJ, 786, 55

Arnett, W. D., Bahcall, J. N., Kirshner, R. P., \& Woosley, S. E. 1989, ARA\&A, 27,629

Barlow, M. J. 1978a, MNRAS, 183, 397

Barlow, M. J. 1978b, MNRAS, 183, 367

Barlow, M. J., Krause, O., Swinyard, B. M., et al. 2010, A\&A, 518, L138

Bianchi, S., \& Schneider, R. 2007, MNRAS, 378, 973

Bocchio, M., Jones, A. P., \& Slavin, J. D. 2014, A\&A, 570, A32

Boyer, M. L., Srinivasan, S., Riebel, D., et al. 2012, ApJ, 748, 40

Chieffi, A., \& Limongi, M. 2013, ApJ, 764, 21

Cioffi, D. F., McKee, C. F., \& Bertschinger, E. 1988, ApJ, 334, 252

Cowie, L. L. 1978, ApJ, 225, 887

Dartois, E., Muñoz Caro, G. M., Deboffle, D., Montagnac, G., \& D’Hendecourt, L. 2005, A\&A, 432, 895

de Bennassuti, M., Schneider, R., Valiante, R., \& Salvadori, S. 2014, MNRAS, 445, 3039

Del Zanna, L., Bucciantini, N., \& Londrillo, P. 2003, A\&A, 400, 397

Dell'Agli, F., Ventura, P., Schneider, R., et al. 2015, MNRAS, 447, 2992

Draine, B. T., \& Hao, L. 2002, ApJ, 569, 780

Draine, B. T., \& Salpeter, E. E. 1979a, ApJ, 231, 438

Draine, B. T., \& Salpeter, E. E. 1979b, ApJ, 231, 77

Dunne, L., Maddox, S. J., Ivison, R. J., et al. 2009, MNRAS, 394, 1307

Dwek, E., \& Arendt, R. G. 2015, ApJ, 810, 75

Dwek, E., \& Scalo, J. M. 1980, ApJ, 239, 193

Eriksen, K. A., Arnett, D., McCarthy, D. W., \& Young, P. 2009, ApJ, 697, 29
Figiel, P., Rozmus, M., \& Smuk, B. 2011, J. Achievements in Materials and Manufacturing Engineering, 48, 1

Gall, C., Hjorth, J., \& Andersen, A. C. 2011, A\&ARv, 19, 43

Gehrz, R. 1989, in Interstellar Dust, eds. L. J. Allamandola, \& A. G. G. M Tielens, IAU Symp., 135, 445

Gomez, H. 2013, in Proc. The Life Cycle of Dust in the Universe: Observations, Theory, and Laboratory Experiments (LCDU2013), 18-22 November, 2013. Taipei, Taiwan, 146

Gomez, H. 2014, Nature, 511, 296

Gomez, H. L., Krause, O., Barlow, M. J., et al. 2012, ApJ, 760, 96 Gordon, K. D., Roman-Duval, J., Bot, C., et al. 2014, ApJ, 797, 85

Guhathakurta, P., \& Draine, B. T. 1989, ApJ, 345, 230

Guillet, V. 2008, Ph.D. Thesis, Université Paris Sud, France

Hwang, U., \& Laming, J. M. 2009, ApJ, 703, 883

Hwang, U., \& Laming, J. M. 2012, ApJ, 746, 130

Indebetouw, R., Matsuura, M., Dwek, E., et al. 2014, ApJ, 782, L2

Inoue, T., Shimoda, J., Ohira, Y., \& Yamazaki, R. 2013, ApJ, 772, L20

Jones, A. P., \& Nuth, J. A. 2011, A\&A, 530, A44

Jones, A. P., \& Tielens, A. G. G. M. 1994, in The Diffuse Interstellar Bands, Proc. Symp., Boulder, Colorado (May 16-19 1994), 79

Jones, A. P., Tielens, A. G. G. M., Hollenbach, D. J., \& McKee, C. F. 1994, ApJ, 433, 797

Jones, A. P., Tielens, A. G. G. M., \& Hollenbach, D. J. 1996, ApJ, 469, 740

Jurac, S., Johnson, R. E., \& Donn, B. 1998, ApJ, 503, 247

Krause, O., Birkmann, S. M., Usuda, T., et al. 2008, Science, 320, 1195

Krzyzanowski, M., Beynon, J. H., \& Farrugia, D. 2010, Oxide Scale Behavior in High Temperature Metal Processing (Wiley)

Lakićević, M., van Loon, J. T., Patat, F., Staveley-Smith, L., \& Zanardo, G. 2011, A\&A, 532, L8

Lakićević, M., van Loon, J. T., Meixner, M., et al. 2015, ApJ, 799, 50

Laming, J. M., \& Hwang, U. 2003, ApJ, 597, 347

Lee, Y.-H., Koo, B.-C., Moon, D.-S., \& Lee, J.-J. 2015, ApJ, 808, 98

Li, W., Chornock, R., Leaman, J., et al. 2011, MNRAS, 412, 1473

Limongi, M., \& Chieffi, A. 2006, ApJ, 647, 483

MacAlpine, G. M., \& Satterfield, T. J. 2008, AJ, 136, 2152

Mancini, M., Schneider, R., Graziani, L., et al. 2015, MNRAS, 451, 70

Marsh, S. P. 1980, LASL Hugoniot shock data, Tech. rep. (Berkeley: Univ. California Press)

Matsuura, M., Barlow, M. J., Zijlstra, A. A., et al. 2009, MNRAS, 396, 918

Matsuura, M., Dwek, E., Meixner, M., et al. 2011, Science, 333, 1258

Matsuura, M., Woods, P. M., \& Owen, P. J. 2013, MNRAS, 429, 2527

Matsuura, M., Dwek, E., Barlow, M. J., et al. 2015, ApJ, 800, 50

Mattsson, L., Gomez, H. L., Andersen, A. C., \& Matsuura, M. 2015, MNRAS, 449, 4079

May, P. W., Pineau des Forêts, G., Flower, D. R., et al. 2000, MNRAS, 318, 809

McKee, C. F., Hollenbach, D. J., Seab, G. C., \& Tielens, A. G. G. M. 1987, ApJ, 318,674

McQueen, R. G., Marsh, S. P., \& Fritz, J. N. 1967, J. Geophys. Res., 72, 4999

Melosh, H. J. 2007, Meteoritics 42, 2079

Micelotta, E. R., Jones, A. P., \& Tielens, A. G. G. M. 2010a, A\&A, 510, A36

Micelotta, E. R., Jones, A. P., \& Tielens, A. G. G. M. 2010b, A\&A, 510, A37

Michałowski, M. J. 2015, A\&A, 577, 80

Nomoto, K., Shigeyama, T., Kumagai, S., Yamaoka, H., \& Suzuki, T. 1994, in Supernovae, eds. S. A. Bludman, R. Mochkovitch, \& J. Zinn-Justin, 489

Nomoto, K., Tominaga, N., Umeda, H., Kobayashi, C., \& Maeda, K. 2006, Nucl. Phys. A, 777, 424

Nozawa, T., Kozasa, T., \& Habe, A. 2006, ApJ, 648, 435

Nozawa, T., Kozasa, T., Habe, A., et al. 2007, ApJ, 666, 955

Olofsson, H., Maercker, M., Eriksson, K., Gustafsson, B., \& Schöier, F. 2010, A\&A, 515, A27

Otsuka, M., van Loon, J. T., Long, K. S., et al. 2010, A\&A, 518, L139

Owen, P. J., \& Barlow, M. J. 2015, ApJ, 801, 141

Park, S., Hughes, J. P., Slane, P. O., et al. 2012, ApJ, 748, 117

Petersen, K. E. 1978, IEEE Transactions on Electron Devices, 25, 1241

Patnaude, D. J., \& Fesen, R. A. 2009, ApJ, 697, 535

Raymond, J. C., Cox, D. P., \& Smith, B. W. 1976, ApJ, 204, 290

Rho, J., Kozasa, T., Reach, W. T., et al. 2008, ApJ, 673, 271

Riebel, D., Srinivasan, S., Sargent, B., \& Meixner, M. 2012, ApJ, 753, 71

Rowlands, K., Gomez, H. L., Dunne, L., et al. 2014, MNRAS, 441, 1040

Schneider, R., Valiante, R., Ventura, P., et al. 2014, MNRAS, 442, 1440

Schure, K. M., \& Bell, A. R. 2014, MNRAS, 437, 2802

Seab, C. G., \& Shull, J. M. 1983, ApJ, 275, 652

Sedov L. I. 1969, Similarity and Dimensional Methods in Mechanics (New York: Academic)

Serra Díaz-Cano, L., \& Jones, A. P. 2008, A\&A, 492, 127

Shull, J. M. 1978, ApJ, 226, 858

Slavin, J. D., Dwek, E., \& Jones, A. P. 2015, ApJ, 803, 7 
Smith, N. 2013, MNRAS, 434, 102

Taylor, G. 1950, Roy. Soc. London Proc. Ser. A, 201, 175

Ventura, P., Criscienzo, M. D., Schneider, R., et al. 2012a, MNRAS, 424,

Tielens, A. G. G. M., McKee, C. F., Seab, C. G., \& Hollenbach, D. J. 1994, ApJ, 431, 321

Truelove, J. K., \& McKee, C. F. 1999, ApJS, 120, 299

Turcotte, D. L., \& Schubert, G. 2014, Geodynamics (Cambridge University Press)

Valiante, R., Schneider, R., Bianchi, S., \& Andersen, A. C. 2009, MNRAS, 397, 1661

Valiante, R., Schneider, R., Salvadori, S., \& Bianchi, S. 2011, MNRAS, 416 1916

Valiante, R., Schneider, R., Salvadori, S., \& Gallerani, S. 2014, MNRAS, 444, 2442

van der Swaluw, E., Achterberg, A., Gallant, Y. A., \& Tóth, G. 2001, A\&A, 380, 309 2345

$$
2345
$$

Ventura, P., di Criscienzo, M., Schneider, R., et al. 2012b, MNRAS, 420, 1442

Watson, D., Christensen, L., Knudsen, K. K., et al. 2015, Nature, 519

Weigelt, G., Balega, Y. Y., Blöcker, T., et al. 2002, A\&A, 392, 131

Wesson, R., Barlow, M. J., Matsuura, M., \& Ercolano, B. 2015, MNRAS, 446, 2089

Woitke, P. 2006, A\&A, 452, 537

Zhou, W., \& Wang, Z. L. 2007, Scanning Microscopy for Nanotechnology: Techniques and Applications (Springer)

Zhukovska, S., Gail, H.-P., \& Trieloff, M. 2008, A\&A, 479, 453

Ziegler, J. F., Biersack, J. P., \& Littmark, U. 1985, Stopping and Range of Ions in Matter, version 2013, www.srim.org
Ventura, P., Dell'Agli, F., Schneider, R., et al. 2014, MNRAS, 439, 977 
M. Bocchio et al.: Dust grains from the heart of supernovae

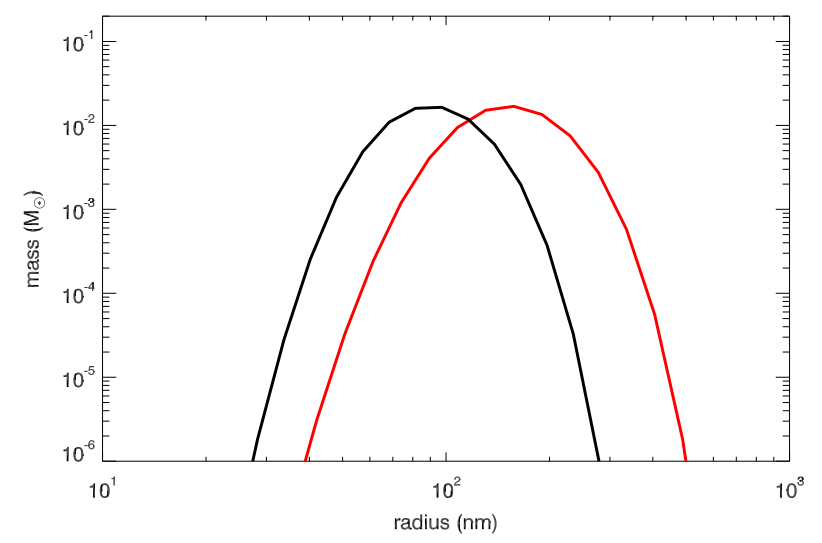

Fig. A.1. Dust mass distribution assuming graphitic-type $(\rho=$ $2.2 \mathrm{~g} \mathrm{~cm}^{-3}$, black line) or amorphous carbon $\left(\rho=1.6 \mathrm{~g} \mathrm{~cm}^{-3}\right.$, red line $)$ grains.

\section{Appendix A: Carbonaceous grain density}

Carbonaceous grains formed in $\mathrm{SNe}$ exhibit a wide range of density $\left(1.6\right.$ to $\left.2.2 \mathrm{~g} \mathrm{~cm}^{-3}\right)$, as is revealed by various measurements from presolar grains (e.g. Amari et al. 2005, 2014). We assumed graphitic-type grains with a density of $2.2 \mathrm{~g} \mathrm{~cm}^{-3}$. In this appendix we consider low-density $\left(\rho=1.6 \mathrm{~g} \mathrm{~cm}^{-3}\right)$ amorphous carbon grains and show the effect of this change on the amount of carbonaceous dust surviving the explosion of a SN.

We considered as an example SN 1987A and reran the dust formation model, lowering the density of carbonaceous grains. In Fig. A.1 we show the initial dust mass distribution for graphitic-type $\left(\rho=2.2 \mathrm{~g} \mathrm{~cm}^{-3}\right.$, black line) and for lowdensity amorphous carbon grains $\left(\rho=1.6 \mathrm{~g} \mathrm{~cm}^{-3}\right.$, red line). While the total carbonaceous dust mass formed in the two cases is very similar $\left(M_{\mathrm{TOT}}=7.15 \times 10^{-2} M_{\odot}\right.$ and $7.02 \times$ $10^{-2} M_{\odot}$ for high- and low-density carbon grains, respectively), the mass distribution for a lower density is shifted towards larger grains ( $a_{0}$ is shifted from $\sim 90 \mathrm{~nm}$ to $\sim 160 \mathrm{~nm}$ for low-density grains).

We then updated the GRASH_Rev code taking into account the carbonaceous grain processing described by Bocchio et al. (2014). In particular, the lower density of the material shows its strongest effect on the sputtering yield, which is enhanced by up to an order of magnitude over a wide range of energies and grain sizes (see Figs. 3 and 4 in Bocchio et al. 2014). However, the mass distribution of low-density carbon grains is shifted towards larger grains, which are therefore less affected by sputtering than smaller, higher-density grains. This cancels out the enhancement of the sputtering yield. The resulting mass of carbon dust surviving the explosion of SN 1987A is then enhanced by a factor $\sim 3$ from $M_{\mathrm{AC}}=8.5 \times 10^{-3} M_{\odot}$ to $2.5 \times 10^{-2} M_{\odot}$.

\section{Appendix B: Size-dependent sputtering}

With the use of the Stopping and Range of Ions in Matter software $^{2}$ (SRIM, Ziegler et al. 1985), it is possible to simulate the sputtering of targets of different materials as they are hit by projectile ions. The simulations provide both the sputtering yield (for a semi-infinite target), $Y_{\infty}$, and the position $(x, y$ and

\footnotetext{
2 SRIM is a collection of software packages that calculates the transport of ions in matter, calibrating the results on the available experimental data.
}

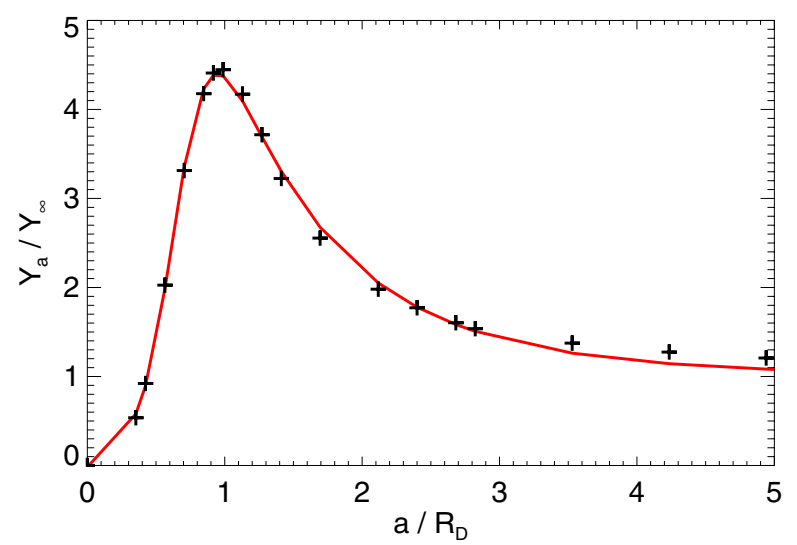

Fig. B.1. Ratio $Y_{\mathrm{a}} / Y_{\infty}$ as a function of $x=a / R_{\mathrm{D}}$ for graphitic-type target material and for impinging He ions. Plus signs represent results from SRIM simulations, while the red solid line indicates the analytical approximation.

$z$ coordinates) of the impinging ions after their interaction with the target.

The target materials and densities used in GRASH_rev are listed in Table B.1. We considered He ions with an energy of $1 \mathrm{keV}$ as projectiles. To estimate the position of the displaced target atoms, we followed the same approximation as adopted by Serra Díaz-Cano \& Jones (2008): a projectile loses most of its energy at a depth of $R_{\mathrm{D}} \sim 0.7 R_{\mathrm{p}}$. We then assumed that target atoms are displaced to a distance of $R_{\mathrm{D}}$ from their original position. In this way, we approximately reconstructed the distribution of displaced target atoms.

We simulated the bombardment of $n_{\text {TOT }}=10^{6}$ ions for each material. At the end of the simulation we then have the $3 \mathrm{D}$ coordinates of the impinging ions and targets. We considered different grain radii, $a$, and counted the number of impinging ions, $n_{\mathrm{i}}$, that are enclosed in a sphere of radius $2 a$ around the grain centre. In this way, we only account for ions that triggered a collisional cascade in the target and exclude transmitted ions. Furthermore, we counted the number of targets, $n_{\mathrm{t}}$, whose position is outside the grain volume and that are associated to ions that triggered collisional cascades in the target. We then estimated the correction to the sputtering yield due to the effect of the grain size as

$Y_{\mathrm{a}}=\left(\frac{n_{\mathrm{i}}-n_{\mathrm{t}}}{n_{\mathrm{TOT}}} Y_{\infty}+\frac{n_{\mathrm{t}}}{n_{\mathrm{TOT}}}\right)$.

In Fig. B. 1 we show the resulting ratio $f(x)=Y_{\mathrm{a}} / Y_{\infty}$ as a function of $x=a / R_{\mathrm{D}}$ (plus signs) for graphitic-type grains. We then fit our results (red line) with the parametric function in Eq. (21). The parameters resulting from the fit are reported in Table 3 for all the materials.

\section{Appendix C: Shattering and vapourisation parameters}

Shattering and vapourisation parameters were calculated following the method by Tielens et al. (1994) and Jones et al. (1996) and based upon material properties found in the literature. The critical energy density for vapourisation, $\epsilon_{\mathrm{th}, \mathrm{v}}$, was calculated starting from the surface binding energy, $E_{\mathrm{b}}$, as follows:

$\epsilon_{\mathrm{th}, \mathrm{v}}=2 \frac{E_{\mathrm{b}}}{m_{\mathrm{p}} M}$, 
Table B.1. Grain density and critical parameters for shattering and vapourisation for all the grain materials considered in the model.

\begin{tabular}{|c|c|c|c|c|c|c|}
\hline Parameter & $\mathrm{Al}_{2} \mathrm{O}_{3}$ & $\mathrm{Fe}_{3} \mathrm{O}_{4}$ & $\mathrm{MgSiO}_{3}$ & $\mathrm{Mg}_{2} \mathrm{SiO}_{4}$ & $\mathrm{AC}$ & $\mathrm{SiO}_{2}$ \\
\hline$\rho_{0}\left[\mathrm{~g} \mathrm{~cm}^{-3}\right]$ & $3.83^{a}$ & $5.12^{a}$ & $3.18^{b}$ & $3.32^{b}$ & 2.2 & $2.65^{c}$ \\
\hline$c_{0}\left[\mathrm{~km} \mathrm{~s}^{-2}\right]$ & $6.91^{a}$ & $6.7^{a}$ & $6.0^{b}$ & $6.0^{b}$ & 1.8 & $3.68^{3}$ \\
\hline$s$ & $1.44^{a}$ & $1.36^{a}$ & $1.13^{b}$ & $0.86^{b}$ & 1.23 & $2.12^{c}$ \\
\hline \multicolumn{7}{|c|}{ Shattering } \\
\hline$\epsilon_{\mathrm{th}}^{\mathrm{sh}}\left[\times 10^{10} \mathrm{erg} \mathrm{g}^{-1}\right]$ & 32.4 & 6.13 & 105 & 105 & 0.08 & 6.31 \\
\hline$P^{\text {sh }}\left[\times 10^{11}\right.$ dyn cm $\left.^{-2}\right]$ & $37.0^{d}$ & $20.8^{e}$ & $9.0^{f}$ & $9.0^{f}$ & 0.2 & $6.0^{g}$ \\
\hline$v_{\text {th }}^{\text {th }}\left[\mathrm{km} \mathrm{s}^{-1}\right]$ & 16.1 & 7.0 & 28.9 & 28.9 & 0.9 & 7.1 \\
\hline \multicolumn{7}{|c|}{ Vapourisation } \\
\hline$\epsilon_{\mathrm{th}}^{\mathrm{v}}\left[\times 10^{10} \mathrm{erg} \mathrm{g}^{-1}\right]$ & $8.1^{h}$ & $9.6^{i}$ & $7.7^{j}$ & $7.7^{j}$ & 64.0 & $30.0^{i}$ \\
\hline$P_{\mathrm{th}}^{\mathrm{v}}\left[\times 10^{11} \mathrm{dyn} \mathrm{cm}^{-2}\right]$ & 1.7 & 6.8 & 5.4 & 5.4 & 58.0 & 23.0 \\
\hline$v_{\mathrm{th}}^{\mathrm{v}}\left[\mathrm{km} \mathrm{s}^{-1}\right]$ & 8.1 & 8.8 & 7.8 & 7.8 & 23.0 & 15.0 \\
\hline
\end{tabular}

References. ${ }^{(a)}$ Marsh (1980); ${ }^{(b)}$ McQueen et al. (1967); ${ }^{(c)}$ Melosh (2007); ${ }^{(d)}$ Figiel et al. (2011); ${ }^{(e)}$ Krzyzanowski et al. (2010); ${ }^{(f)}$ Turcotte \& Schubert (2014); ${ }^{(g)}$ Petersen (1978); ${ }^{(h)}$ Zhou \& Wang (2007); ${ }^{(i)}$ JANAF tables (http://kinetics.nist.gov/janaf/); ${ }^{(j)}$ May et al. (2000).

where $m_{\mathrm{p}}$ is the proton mass and $M$ is the molar mass of the considered material. Then, the critical relative velocity between grains to set the vapourisation process is given by

$v_{\mathrm{th}, \mathrm{v}}=2\left(2 \epsilon_{\mathrm{th}}^{\mathrm{v}}\right)^{2}$

and the corresponding pressure in the shocked solid is obtained as (Tielens et al. 1994)

$P_{\mathrm{th}, \mathrm{v}}=\frac{\rho_{0} v_{\mathrm{th}, \mathrm{v}}\left(v_{\mathrm{th}, \mathrm{v}}-c_{0}\right)}{s}$.

The critical pressure, $P_{\text {th,sh }}$, above which grains undergo fragmentation was assumed equal to the Young's modulus of the material. The critical velocity for the onset of crater formation is given by (Jones et al. 1994)

$v_{\mathrm{th}, \mathrm{sh}}=\sqrt{4 / 3} \frac{P_{\mathrm{th}, \mathrm{sh}}}{\rho_{0} c_{0}}$,

and the critical energy density results to be

$\epsilon_{\mathrm{th}, \mathrm{sh}}=\frac{1}{2}\left(\frac{v_{\mathrm{th}, \mathrm{sh}}}{2}\right)^{2}$.

In Table B.1 we report all the values for vapourisation and shattering critical parameters that we adopted in GRASH_rev. 